\title{
Circular and noncircular nearly horizon-skimming orbits in Kerr spacetimes
}

\author{
Enrico Barausse* \\ SISSA, International School for Advanced Studies and INFN, Via Beirut 2, 34014 Trieste, Italy
}

Scott A. Hughes

Department of Physics and MIT Kavli Institute, MIT, 77 Massachusetts Ave., Cambridge, Massachusetts 02139, USA

Luciano Rezzolla

Max-Planck-Institut für Gravitationsphysik, Albert-Einstein-Institut, 14476 Potsdam, Germany and Department of Physics, Louisiana State University, Baton Rouge, Louisiana 70803, USA

(Received 3 April 2007; published 8 August 2007)

\begin{abstract}
We have performed a detailed analysis of orbital motion in the vicinity of a nearly extremal Kerr black hole. For very rapidly rotating black holes — spin parameter $a \equiv J / M>0.9524 M$ — we have found a class of very strong-field eccentric orbits whose orbital angular momentum $L_{z}$ increases with the orbit's inclination with respect to the equatorial plane, while keeping latus rectum and eccentricity fixed. This behavior is in contrast with Newtonian intuition, and is in fact opposite to the normal behavior of black hole orbits. Such behavior was noted previously for circular orbits; since it only applies to orbits very close to the black hole, they were named "nearly horizon-skimming orbits." Our current analysis generalizes this result, mapping out the full generic (inclined and eccentric) family of nearly horizonskimming orbits. The earlier work on circular orbits reported that, under gravitational radiation emission, nearly horizon-skimming orbits exhibit unusual inspiral, tending to evolve to smaller orbit inclination, toward prograde equatorial configuration. Normal orbits, by contrast, always demonstrate slowly growing orbit inclination-orbits evolve toward the retrograde equatorial configuration. Using up-to-date Teukolsky-based fluxes, we have concluded that the earlier result was incorrect-all circular orbits, including nearly horizon-skimming ones, exhibit growing orbit inclination under radiative backreaction. Using kludge fluxes based on a Post-Newtonian expansion corrected with fits to circular and to equatorial Teukolsky-based fluxes, we argue that the inclination grows also for eccentric nearly horizon-skimming orbits. We also find that the inclination change is, in any case, very small. As such, we conclude that these orbits are not likely to have a clear and peculiar imprint on the gravitational waveforms expected to be measured by the space-based detector LISA.
\end{abstract}

DOI: $10.1103 /$ PhysRevD.76.044007

PACS numbers: 04.30. $-\mathrm{w}$

\section{INTRODUCTION}

The space-based gravitational-wave detector LISA [1] will be a unique tool to probe the nature of supermassive black holes (SMBHs), making it possible to map in detail their spacetimes. This goal is expected to be achieved by observing gravitational waves emitted by compact stars or black holes with masses $\mu \approx 1-100 M_{\odot}$ spiraling into the SMBHs which reside in the center of galaxies [2] (particularly the low end of the galactic center black hole mass function, $\left.M \approx 10^{5}-10^{7} M_{\odot}\right)$. Such events are known as extreme mass ratio inspirals (EMRIs). Current wisdom suggests that several tens to perhaps of order a thousand such events could be measured per year by LISA [3].

Though the distribution of spins for observed astrophysical black holes is not very well understood at present, very rapid spin is certainly plausible, as accretion tends to spinup SMBHs [4]. Most models for quasi-periodic oscillations (QPOs) suggest this is indeed the case in all low-mass $\mathrm{X}$ ray binaries for which data is available [5]. On the other

*barausse@sissa.it hand, continuum spectral fitting of some high-mass x-ray binaries indicates that modest spins (spin parameter $\left.a / M \equiv J / M^{2} \sim 0.6-0.8\right)$ are likewise plausible [6]. The continuum-fit technique does find an extremely high spin of $a / M \geq 0.98$ for the galactic "microquasar" GRS1915 + 105 [7]. This argues for a wide variety of possible spins, depending on the detailed birth and growth history of a given black hole.

In the mass range corresponding to black holes in galactic centers, measurements of the broad iron $\mathrm{K} \alpha$ emission line in active galactic nuclei suggest that SMBHs can be very rapidly rotating (see Ref. [8] for a recent review). For instance, in the case of MCG-6-30-15, for which highly accurate observations are available, $a$ has been found to be larger than $0.987 \mathrm{M}$ at $90 \%$ confidence [9]. Because gravitational waves from EMRIs are expected to yield a very precise determination of the spins of SMBHs [10], it is interesting to investigate whether EMRIs around very rapidly rotating black holes may possess peculiar features which would be observable by LISA. Should such features exist, they would provide unambiguous information on the spin of SMBHs and thus on the mechanisms leading to their formation [11]. 
For extremal Kerr black holes $(a=M)$, the existence of a special class of "circular" orbits was pointed out long ago by Wilkins [12], who named them "horizonskimming" orbits. (Circular here means that the orbits are of constant Boyer-Lindquist coordinate radius $r$.) These orbits have varying inclination angle with respect to the equatorial plane and have the same coordinate radius as the horizon, $r=M$. Despite this seemingly hazardous location, it can be shown that all these $r=M$ orbits have finite separation from one another and from the event horizon [13]. Their somewhat pathological description is due to a singularity in the Boyer-Lindquist coordinates, which collapses a finite span of the spacetime into $r=M$.

Besides being circular and "horizon-skimming," these orbits also show peculiar behavior in their relation of angular momentum to inclination. In Newtonian gravity, a generic orbit has $L_{z}=|\boldsymbol{L}| \cos \iota$, where $\iota$ is the inclination angle relative to the equatorial plane (going from $\iota=0$ for equatorial prograde orbits to $\iota=\pi$ for equatorial retrograde orbits, passing through $\iota=\pi / 2$ for polar orbits), and $\boldsymbol{L}$ is the orbital angular momentum vector. As a result, $\partial L_{z}(r, \iota) / \partial \iota<0$, and the angular momentum in the $z$-direction always decreases with increasing inclination if the orbit's radius is kept constant. This intuitively reasonable decrease of $L_{z}$ with $\iota$ is seen for almost all black hole orbits as well. Horizon-skimming orbits, by contrast, exhibit exactly the opposite behavior: $L_{z}$ increases with inclination angle.

Reference [14] asked whether the behavior $\partial L_{z} / \partial \iota>0$ could be extended to a broader class of circular orbits than just those at the radius $r=M$ for the spin value $a=M$. It was found that this condition is indeed more general, and extended over a range of radius from the "innermost stable circular orbit" to $r \simeq 1.8 M$ for black holes with $a>$ $0.9524 M$. Orbits that show this property have been named "nearly horizon-skimming." The Newtonian behavior $\partial L_{z}(r, \iota) / \partial \iota<0$ is recovered for all orbits at $r \gtrsim 1.8 M$ [14].

A qualitative understanding of this behavior comes from recalling that very close to the black hole all physical processes become "locked" to the hole's event horizon [15], with the orbital motion of point particles coupling to the horizon's spin. This locking dominates the "Keplerian" tendency of an orbit to move more quickly at smaller radii, forcing an orbiting particle to slow down in the innermost orbits. Locking is particularly strong for the most-bound (equatorial) orbits; the least-bound orbits (which have the largest inclination) do not strongly lock to the black hole's spin until they have very nearly reached the innermost orbit [14]. The property $\partial L_{z}(r, \iota) / \partial \iota>0$ reflects the different efficiency of nearly horizon-skimming orbits to lock with the horizon.

Reference [14] argued that this behavior could have observational consequences. It is well-known that the inclination angle of an inspiraling body generally increases due to gravitational-wave emission [16,17]. Since $d L_{z} / d t<0$ because of the positive angular momentum carried away by the gravitational waves, and since "normal" orbits have $\partial L_{z} / \partial \iota<0$, one would expect $d \iota / d t>$ 0 . However, if during an evolution $\partial L_{z} / \partial \iota$ switches sign, then $d \iota / d t$ might switch sign as well: An inspiraling body could evolve towards an equatorial orbit, signaling the presence of an "almost-extremal" Kerr black hole.

It should be emphasized that this argument is not rigorous. In particular, one needs to consider the joint evolution of orbital radius and inclination angle; and, one must include the dependence of these two quantities on orbital energy as well as angular momentum. ${ }^{1}$ As such, $d \iota / d t$ depends not only on $d L_{z} / d t$ and $\partial L_{z} / \partial \iota$, but also on $d E / d t, \partial E / \partial \iota, \partial E / \partial r$ and $\partial L_{z} / \partial r$.

In this sense, the argument made in Ref. [14] should be seen as claiming that the contribution coming from $d L_{z} / d t$ and $\partial L_{z} / \partial \iota$ are simply the dominant ones. Using the numerical code described in [17] to compute the fluxes $d L_{z} / d t$ and $d E / d t$, it was then found that a test particle on a circular orbit passing through the nearly horizonskimming region of a Kerr black hole with $a=0.998 M$ (the value at which a hole's spin tends to be buffered due to photon capture from thin disk accretion [19]) had its inclination angle decreased by $\delta \iota \approx 1^{\circ}-2^{\circ}$ [14] in the adiabatic approximation [20]. It should be noted at this point that the rate of change of inclination angle, $d \iota / d t$, appears as the difference of two relatively small and expensive to compute rates of change (cf. Eq. (3.8) of Ref. [17]). As such, small relative errors in those rates of change can lead to large relative errors in $d \iota / d t$. Finally, in Ref. [14] it was speculated that the decrease could be even larger for eccentric orbits satisfying the condition $\partial L_{z} / \partial \iota>0$, possibly leading to an observable imprint on EMRI gravitational waveforms.

The main purpose of this paper is to extend Ref. [14]'s analysis of nearly horizon-skimming orbits to include the effect of orbital eccentricity, and to thereby test the speculation that there may be an observable imprint on EMRI waveforms of nearly horizon-skimming behavior. In doing so, we have revisited all the calculations of Ref. [14] using a more accurate Teukolsky solver which serves as the engine for the analysis presented in Ref. [21].

We have found that the critical spin value for circular nearly horizon-skimming orbits, $a>0.9524 M$, also delineates a family of eccentric orbits for which the condition $\partial L_{z}(p, e, \iota) / \partial \iota>0$ holds. (More precisely, we consider variation with respect to an angle $\theta_{\text {inc }}$ that is easier to work with in the extreme strong field, but that is easily related to $\iota$.) The parameters $p$ and $e$ are the orbit's latus rectum and eccentricity, defined precisely in Sec. II. These

\footnotetext{
${ }^{1}$ In the general case, one must also include the dependence on "Carter's constant" $Q$ [18], the third integral of black hole orbits (described more carefully in Sec. II). For circular orbits, $Q=$ $Q\left(E, L_{z}\right)$ : Knowledge of $E$ and $L_{z}$ completely determines $Q$.
} 
generic nearly horizon-skimming orbits all have $p \lesssim 2 M$, deep in the black hole's extreme strong field.

We next study the evolution of these orbits under gravitational-wave emission in the adiabatic approximation. We first revisited the evolution of circular, nearly horizon-skimming orbits using the improved Teukolsky solver which was used for the analysis of Ref. [21]. The results of this analysis were somewhat surprising: Just as for normal orbits, we found that orbital inclination always increases during inspiral, even in the nearly horizonskimming regime. This is in stark contrast to the claims of Ref. [14]. As noted above, the inclination's rate of change depends on the difference of two expensive and difficult to compute numbers, and thus can be strongly impacted by small relative errors in those numbers. A primary result of this paper is thus to retract the claim of Ref. [14] that an important dynamic signature of the nearly horizon-skimming region is a reversal in the sign of inclination angle evolution: The inclination always grows under gravitational radiation emission.

We next extended this analysis to study the evolution of generic nearly horizon-skimming orbits. The Teukolsky code to which we have direct access can, at this point, only compute the radiated fluxes of energy $E$ and angular momentum $L_{z}$; results for the evolution of the Carter constant $Q$ are just now beginning to be understood [22], and have not yet been incorporated into this code. We instead use "kludge" expressions for $d E / d t, d L_{z} / d t$, and $d Q / d t$ which were inspired by Refs. [23,24]. These expressions are based on post-Newtonian flux formulas, modified in such a way that they fit strong-field radiation reaction results obtained from a Teukolsky integrator; see Ref. [24] for further discussion. Our analysis indicates that, just as in the circular limit, the result $d \iota / d t>0$ holds for generic nearly horizon-skimming orbits. Furthermore, and contrary to the speculation of Ref. [14], we do not find a large amplification of $d \iota / d t$ as orbits are made more eccentric.

Our conclusion is that the nearly horizon-skimming regime, though an interesting curiosity of strong-field orbits of nearly extremal black holes, will not imprint any peculiar observational signature on EMRI waveforms.

The remainder of this paper is organized as follows. In Sec. II, we review the properties of bound stable orbits in Kerr, providing expressions for the constants of motion which we will use in Sec. III to generalize nearly horizonskimming orbits to the noncircular case. In Sec. IV, we study the evolution of the inclination angle for circular nearly horizon-skimming orbits using Teukolsky-based fluxes; in Sec. V we do the same for noncircular orbits, using kludge fluxes. We present and discuss our detailed conclusions in Sec. VI. The fits and post-Newtonian fluxes used for the kludge fluxes are presented in the Appendix. Throughout the paper we have used units in which $G=$ $c=1$.

\section{BOUND STABLE ORBITS IN KERR SPACETIMES}

The line element of a Kerr spacetime, written in BoyerLindquist coordinates reads [25]

$$
\begin{aligned}
d s^{2}= & -\left(1-\frac{2 M r}{\Sigma}\right) d t^{2}+\frac{\Sigma}{\Delta} d r^{2}+\Sigma d \theta^{2}+\left(r^{2}+a^{2}\right. \\
& \left.+\frac{2 M a^{2} r}{\Sigma} \sin ^{2} \theta\right) \sin ^{2} \theta d \phi^{2}-\frac{4 M a r}{\Sigma} \sin ^{2} \theta d t d \phi,
\end{aligned}
$$

where

$$
\Sigma \equiv r^{2}+a^{2} \cos ^{2} \theta, \quad \Delta \equiv r^{2}-2 M r+a^{2} .
$$

Up to initial conditions, geodesics can then be labeled by four constants of motion: the mass $\mu$ of the test particle, its energy $E$ and angular momentum $L_{z}$ as measured by an observer at infinity and the Carter constant $Q$ [18]. The presence of these four conserved quantities makes the geodesic equations separable in Boyer-Lindquist coordinates. Introducing the Carter time $\lambda$, defined by

$$
\frac{d \tau}{d \lambda} \equiv \Sigma,
$$

the geodesic equations become

$$
\begin{array}{ll}
\left(\mu \frac{d r}{d \lambda}\right)^{2}=V_{r}(r), & \mu \frac{d t}{d \lambda}=V_{t}(r, \theta), \\
\left(\mu \frac{d \theta}{d \lambda}\right)^{2}=V_{\theta}(\theta), & \mu \frac{d \phi}{d \lambda}=V_{\phi}(r, \theta),
\end{array}
$$

with

$$
\begin{aligned}
V_{t}(r, \theta) & \equiv E\left(\frac{\varpi^{4}}{\Delta}-a^{2} \sin ^{2} \theta\right)+a L_{z}\left(1-\frac{\varpi^{2}}{\Delta}\right), \\
V_{r}(r) & \equiv\left(E \varpi^{2}-a L_{z}\right)^{2}-\Delta\left[\mu^{2} r^{2}+\left(L_{z}-a E\right)^{2}+Q\right], \\
V_{\theta}(\theta) & \equiv Q-L_{z}^{2} \cot ^{2} \theta-a^{2}\left(\mu^{2}-E^{2}\right) \cos ^{2} \theta, \\
V_{\phi}(r, \theta) & \equiv L_{z} \csc ^{2} \theta+a E\left(\frac{\varpi^{2}}{\Delta}-1\right)-\frac{a^{2} L_{z}}{\Delta},
\end{aligned}
$$

where we have defined

$$
\varpi^{2} \equiv r^{2}+a^{2} .
$$

The conserved parameters $E, L_{z}$, and $Q$ can be remapped to other parameters that describe the geometry of the orbit. We have found it useful to describe the orbit in terms of an angle $\theta_{\min }$ - the minimum polar angle reached by the orbit - as well as the latus rectum $p$ and the eccentricity $e$. In the weak-field limit, $p$ and $e$ correspond exactly to the latus rectum and eccentricity used to describe orbits in Newtonian gravity; in the strong field, they are essentially just a convenient remapping of the orbit's apoastron and periastron: 


$$
r_{\text {ap }} \equiv \frac{p}{1-e}, \quad r_{\text {peri }} \equiv \frac{p}{1+e} .
$$

Finally, in much of our analysis, it is useful to refer to

$$
z_{-} \equiv \cos ^{2} \theta_{\min }
$$

rather than to $\theta_{\min }$ directly.

To map $\left(E, L_{z}, Q\right)$ to $\left(p, e, z_{-}\right)$, use Eq. (4) to impose $d r / d \lambda=0$ at $r=r_{\text {ap }}$ and $r=r_{\text {peri }}$, and to impose $d \theta / d \lambda=0$ at $\theta=\theta_{\min }$. (Note that for a circular orbit, $r_{\text {ap }}=r_{\text {peri }}=r_{0}$. In this case, one must apply the rules $d r / d \lambda=0$ and $d^{2} r / d \lambda^{2}=0$ at $r=r_{0}$.) Following this approach, Schmidt [26] was able to derive explicit expressions for $E, L_{z}$ and $Q$ in terms of $p, e$ and $z_{-}$. We now briefly review Schmidt's results.

Let us first introduce the dimensionless quantities

$$
\begin{gathered}
\tilde{E} \equiv E / \mu, \quad \tilde{L}_{z} \equiv L_{z} /(\mu M), \quad \tilde{Q} \equiv Q /(\mu M)^{2}, \\
\tilde{a} \equiv a / M, \quad \tilde{r} \equiv r / M, \quad \tilde{\Delta} \equiv \Delta / M^{2},
\end{gathered}
$$

and the functions

$$
\begin{gathered}
f(\tilde{r}) \equiv \tilde{r}^{4}+\tilde{a}^{2}\left[\tilde{r}(\tilde{r}+2)+z_{-} \tilde{\Delta}\right], \\
g(\tilde{r}) \equiv 2 \tilde{a} \tilde{r}, \\
h(\tilde{r}) \equiv \tilde{r}(\tilde{r}-2)+\frac{z_{-}}{1-z_{-}} \tilde{\Delta}, \\
d(\tilde{r}) \equiv\left(\tilde{r}^{2}+\tilde{a}^{2} z_{-}\right) \tilde{\Delta} .
\end{gathered}
$$

Let us further define the set of functions

$$
\left(f_{1}, g_{1}, h_{1}, d_{1}\right) \equiv \begin{cases}\left(f\left(\tilde{r}_{p}\right), g\left(\tilde{r}_{p}\right), h\left(\tilde{r}_{p}\right), d\left(\tilde{r}_{p}\right)\right) & \text { if } e>0, \\ \left(f\left(\tilde{r}_{0}\right), g\left(\tilde{r}_{0}\right), h\left(\tilde{r}_{0}\right), d\left(\tilde{r}_{0}\right)\right) & \text { if } e=0,\end{cases}
$$

$\left(f_{2}, g_{2}, h_{2}, d_{2}\right) \equiv \begin{cases}\left(f\left(\tilde{r}_{a}\right), g\left(\tilde{r}_{a}\right), h\left(\tilde{r}_{a}\right), d\left(\tilde{r}_{a}\right)\right) & \text { if } e>0 \\ \left(f^{\prime}\left(\tilde{r}_{0}\right), g^{\prime}\left(\tilde{r}_{0}\right), h^{\prime}\left(\tilde{r}_{0}\right), d^{\prime}\left(\tilde{r}_{0}\right)\right) & \text { if } e=0,\end{cases}$

and the determinants

$$
\begin{gathered}
\kappa \equiv d_{1} h_{2}-d_{2} h_{1}, \\
\varepsilon \equiv d_{1} g_{2}-d_{2} g_{1}, \\
\rho \equiv f_{1} h_{2}-f_{2} h_{1}, \\
\eta \equiv f_{1} g_{2}-f_{2} g_{1}, \\
\sigma \equiv g_{1} h_{2}-g_{2} h_{1} .
\end{gathered}
$$

The energy of the particle can then be written

$$
\tilde{E}=\sqrt{\frac{\kappa \rho+2 \epsilon \sigma-2 D \sqrt{\sigma\left(\sigma \epsilon^{2}+\rho \epsilon \kappa-\eta \overline{\kappa^{2}}\right)}}{\rho^{2}+4 \eta \sigma}} .
$$

The parameter $D$ takes the values \pm 1 . The angular momentum is a solution of the system

$$
\begin{aligned}
& f_{1} \tilde{E}^{2}-2 g_{1} \tilde{E} \tilde{L}_{z}-h_{1} \tilde{L}_{z}^{2}-d_{1}=0, \\
& f_{2} \tilde{E}^{2}-2 g_{2} \tilde{E} \tilde{L}_{z}-h_{2} \tilde{L}_{z}^{2}-d_{2}=0 .
\end{aligned}
$$

By eliminating the $\tilde{L}_{z}^{2}$ terms in these equations, one finds the solution

$$
\tilde{L}_{z}=\frac{\rho \tilde{E}^{2}-\kappa}{2 \tilde{E} \sigma}
$$

for the angular momentum. Using $d \theta / d \lambda=0$ at $\theta=\theta_{\min }$, the Carter constant can be written

$$
\tilde{Q}=z_{-}\left[\tilde{a}^{2}\left(1-\tilde{E}^{2}\right)+\frac{\tilde{L}_{z}^{2}}{1-z_{-}}\right] .
$$

Additional constraints on $p, e, z_{-}$are needed for the orbits to be stable. Inspection of Eq. (4) shows that an eccentric orbit is stable only if

$$
\frac{\partial V_{r}}{\partial r}\left(r_{\text {peri }}\right)>0 .
$$

It is marginally stable if $\partial V_{r} / \partial r=0$ at $r=r_{\text {peri }}$. Similarly, the stability condition for circular orbits is

$$
\frac{\partial^{2} V_{r}}{\partial r^{2}}\left(r_{0}\right)<0
$$

marginally stable orbits are set by $\partial^{2} V_{r} / \partial r^{2}=0$ at $r=r_{0}$.

Finally, we note that one can massage the above solutions for the conserved orbital quantities of bound stable orbits to rewrite the solution for $\tilde{L}_{z}$ as

$$
\tilde{L}_{z}=-\frac{g_{1} \tilde{E}}{h_{1}}+\frac{D}{h_{1}} \sqrt{g_{1}^{2} \tilde{E}^{2}+\left(f_{1} \tilde{E}^{2}-d_{1}\right) h_{1}} .
$$

From this solution, we see that it is quite natural to refer to orbits with $D=1$ as prograde and to orbits with $D=-1$ as retrograde. Note also that Eq. (29) is a more useful form than the corresponding expression, Eq. (A4), of Ref. [21]. In that expression, the factor $1 / h_{1}$ has been squared and moved inside the square root. This obscures the fact that $h_{1}$ changes sign for very strong field orbits. Differences between Eq. (29) and Eq. (A4) of [21] are apparent for $a \gtrsim$ 0.835 , although only for orbits close to the separatrix (i.e., the surface in the space of parameters $(p, e, \iota)$ where marginally stable bound orbits lie). 


\section{NONCIRCULAR NEARLY HORIZON- SKIMMING ORBITS}

With explicit expressions for $E, L_{z}$ and $Q$ as functions of $p, e$ and $z_{-}$, we now examine how to generalize the condition $\partial L_{z}(r, \iota) / \partial \iota>0$, which defined circular nearly horizon-skimming orbits in Ref. [14], to encompass the noncircular case. We recall that the inclination angle $\iota$ is defined as [14]

$$
\cos \iota=\frac{L_{z}}{\sqrt{Q+L_{z}^{2}}} .
$$

Such a definition is not always easy to handle in the case of eccentric orbits. In addition, $\iota$ does not have an obvious physical interpretation (even in the circular limit), but rather was introduced essentially to generalize (at least formally) the definition of inclination for Schwarzschild black hole orbits. In that case, one has $Q=L_{x}^{2}+L_{y}^{2}$ and therefore $L_{z}=|\boldsymbol{L}| \cos \boldsymbol{\iota}$.

A more useful definition for the inclination angle in Kerr was introduced in Ref. [21]:

$$
\theta_{\text {inc }}=\frac{\pi}{2}-D \theta_{\text {min }}
$$

where $\theta_{\min }$ is the minimum reached by $\theta$ during the orbital motion. This angle is trivially related to $z_{-}\left(z_{-}=\sin ^{2} \theta_{\text {inc }}\right)$ and ranges from 0 to $\pi / 2$ for prograde orbits and from $\pi / 2$ to $\pi$ for retrograde orbits. It is a simple numerical calculation to convert between $\iota$ and $\theta_{\text {inc }}$; doing so shows that the differences between $\iota$ and $\theta_{\text {inc }}$ are very small, with the two coinciding for $a=0$, and with a difference that is less than $2.6^{\circ}$ for $a=M$ and circular orbits with $r=M$.

Bearing all this in mind, the condition we have adopted to generalize nearly horizon-skimming orbits is

$$
\frac{\partial L_{z}\left(p, e, \theta_{\text {inc }}\right)}{\partial \theta_{\text {inc }}}>0
$$

We have found that certain parts of this calculation, particular the analysis of strong-field geodesic orbits, are best done using the angle $\theta_{\text {inc }}$; other parts are more simply done using the angle $\iota$, particularly the kludge computation of fluxes described in Sec. V. (This is because the kludge fluxes are based on an extension of post-Newtonian formulas to the strong-field regime, and these formulas use $\iota$ for inclination angle.) Accordingly, we often switch back and forth between these two notions of inclination, and in fact present our final results for inclination evolution using both $d \iota / d t$ and $d \theta_{\text {inc }} / d t$.

Before mapping out the region corresponding to nearly horizon-skimming orbits, it is useful to examine stable orbits more generally in the strong field of rapidly rotating black holes. We first fix a value for $a$, and then discretize the space of parameters $\left(p, e, \theta_{\text {inc }}\right)$. We next identify the points in this space corresponding to bound stable geodesic orbits. Sufficiently close to the horizon, the bound stable orbits with specified values of $p$ and $e$ have an inclination angle $\theta_{\text {inc }}$ ranging from 0 (equatorial orbit) to a maximum value $\theta_{\mathrm{inc}}^{\max }$. For given $p$ and $e, \theta_{\mathrm{inc}}^{\max }$ defines the separatrix between stable and unstable orbits.

Example separatrices are shown in Fig. 1 for $a=$ $0.998 M$ and $a=M$. This figure shows the behavior of $\theta_{\text {inc }}^{\max }$ as a function of the latus rectum for the different values of the eccentricity indicated by the labels. Note that for $a=0.998 M$ the angle $\theta_{\text {inc }}^{\max }$ eventually goes to zero. This is the general behavior for $a<M$. On the other hand, for an extremal black hole, $a=M, \theta_{\text {inc }}^{\max }$ never goes to zero. The orbits which reside at $r=M$ (the circular limit) are the "horizon-skimming orbits" identified by Wilkins [12]; the $a=M$ separatrix has a similar shape even for eccentric orbits. As expected, we find that for given latus rectum and eccentricity the orbit with $\theta_{\text {inc }}=0$ is the one with the lowest energy $E$ (and hence is the mostbound orbit), whereas the orbit with $\theta_{\text {inc }}=\theta_{\text {inc }}^{\max }$ has the highest $E$ (and is least-bound).

Having mapped out stable orbits in $\left(p, e, \theta_{\text {inc }}\right)$ space, we then computed the partial derivative $\partial L_{z}\left(p, e, \theta_{\text {inc }}\right) / \partial \theta_{\text {inc }}$ and identified the following three overlapping regions:

(i) Region A: The portion of the $(p, e)$ plane for which $\partial L_{z}\left(p, e, \theta_{\text {inc }}\right) / \partial \theta_{\text {inc }}>0$ for $0 \leq \theta_{\text {inc }} \leq \theta_{\text {inc }}^{\max }$. This region is illustrated in Fig. 2 as the area under the heavy solid line and to the left of the dashed-dotted line (green in the online color version).

(ii) Region $\mathrm{B}$ : The portion of the $(p, e)$ plane for which $\left(L_{z}\right)_{\text {most bound }}(p, e)$ is smaller than $\left(L_{z}\right)_{\text {least bound }}(p, e)$. In other words,

$$
L_{z}(p, e, 0)<L_{z}\left(p, e, \theta_{\mathrm{inc}}^{\max }\right)
$$

in Region B. Note that Region B contains Region A. It is illustrated in Fig. 2 as the area under the heavy solid line and to the left of the dotted line (red in the online color version).

(iii) Region C: The portion of the $(p, e)$ plane for which $\partial L_{z}\left(p, e, \theta_{\text {inc }}\right) / \partial \theta_{\text {inc }}>0$ for at least one angle $\theta_{\text {inc }}$ between 0 and $\theta_{\mathrm{inc}}^{\max }$. Region $\mathrm{C}$ contains Region $\mathrm{B}$, and is illustrated in Fig. 2 as the area under the heavy solid line and to the left of the dashed line (blue in the online color version).

Orbits in any of these three regions give possible generalizations of the nearly horizon-skimming circular orbits presented in Ref. [14]. Notice, as illustrated in Fig. 2, that the size of these regions depends rather strongly on the spin of the black hole. All three regions disappear altogether for $a<0.9524 M$ (in agreement with [14]); their sizes grow with $a$, reaching maximal extent for $a=M$. These regions never extend beyond $p \simeq 2 M$.

As we shall see, the difference between these three regions is not terribly important for assessing whether there is a strong signature of the nearly horizon-skimming regime on the inspiral dynamics. As such, it is perhaps most useful to use Region $\mathrm{C}$ as our definition, since it is the most inclusive. 

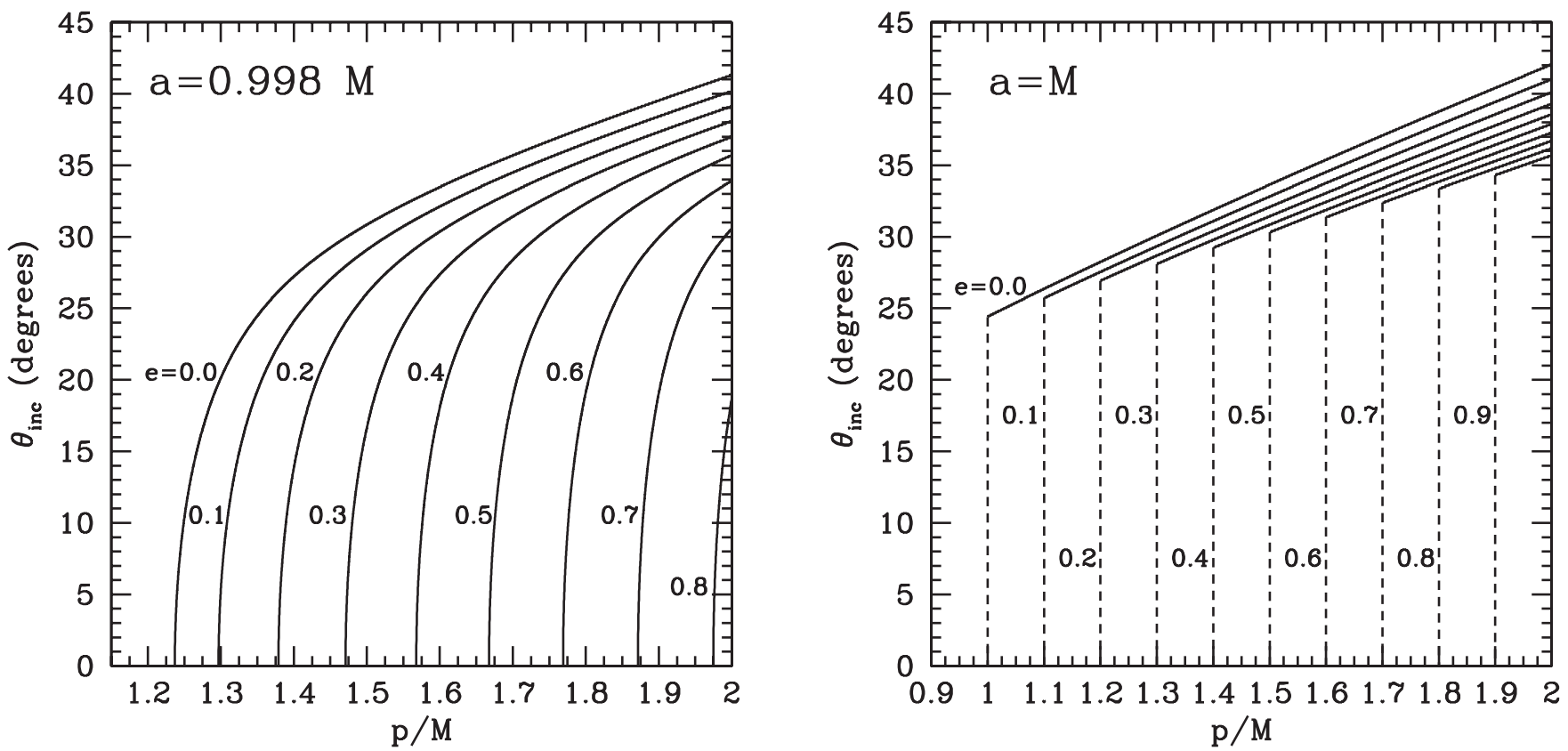

FIG. 1. Left panel: Inclination angles $\theta_{\text {inc }}$ for which bound stable orbits exist for a black hole with spin $a=0.998 M$. The allowed range for $\theta_{\text {inc }}$ goes from $\theta_{\text {inc }}=0$ to the curve corresponding to the eccentricity under consideration, $\theta_{\text {inc }}=\theta_{\text {inc }}^{\max }$. Right panel: Same as the left panel, but for an extremal black hole, $a=M$. Note that in this case $\theta_{\text {inc }}^{\max }$ never reaches zero.
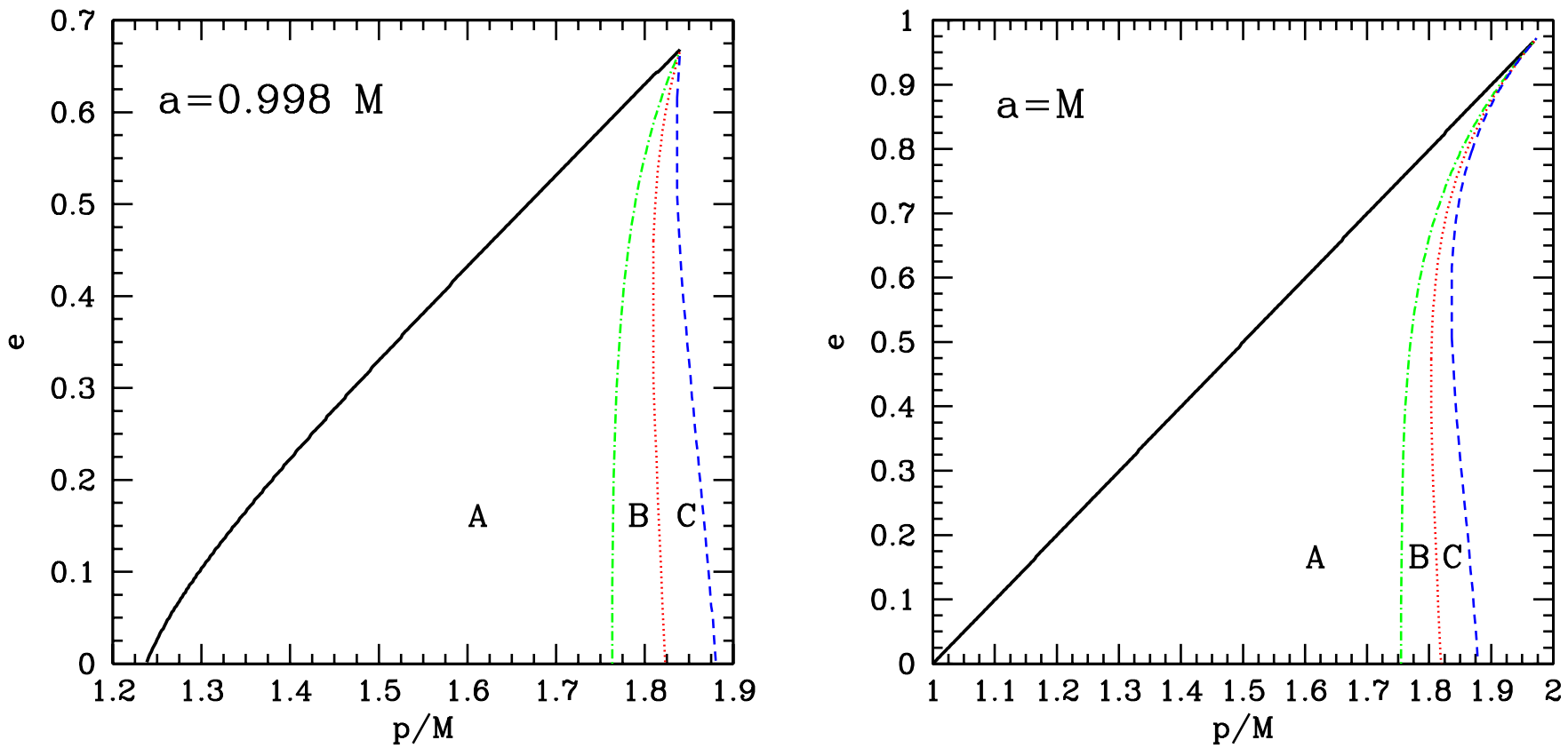

FIG. 2 (color online). Left panel: Noncircular nearly horizon-skimming orbits for $a=0.998 M$. The heavy solid line indicates the separatrix between stable and unstable orbits for equatorial orbits $\left(\iota=\theta_{\text {inc }}=0\right)$. All orbits above and to the left of this line are unstable. The dashed-dotted line (green in the online color version) bounds the region of the $(p, e)$-plane where $\partial L_{z} / \partial \theta_{\text {inc }}>0$ for all allowed inclination angles ("Region A"). All orbits between this line and the separatrix belong to Region A. The dotted line (red in the online color version) bounds the region $\left(L_{z}\right)_{\text {most bound }}<\left(L_{z}\right)_{\text {least bound }}$ ("Region B"). Note that B includes A. The dashed line (blue in the online color version) bounds the region where $\partial L_{z} / \partial \theta_{\text {inc }}>0$ for at least one inclination angle ("Region C"); note that C includes B. All three of these regions are candidate generalizations of the notion of nearly horizon-skimming orbits. Right panel: Same as the left panel, but for the extreme spin case, $a=M$. In this case the separatrix between stable and unstable equatorial orbits is given by the line $p / M=1+e$. 


\section{EVOLUTION OF $\theta_{\text {inc }}$ : CIRCULAR ORBITS}

To ascertain whether nearly horizon-skimming orbits can affect an EMRI in such a way as to leave a clear imprint in the gravitational-wave signal, we have studied the time evolution of the inclination angle $\theta_{\text {inc }}$. To this purpose we have used the so-called adiabatic approximation [20], in which the infalling body moves along a geodesic with slowly changing parameters. The evolution of the orbital parameters is computed using the timeaveraged fluxes $d E / d t, d L_{z} / d t$ and $d Q / d t$ due to gravitational-wave emission ("radiation reaction"). As discussed in Sec. II, $E, L_{z}$ and $Q$ can be expressed in terms of $p, e$, and $\theta_{\text {inc }}$. Given rates of change of $E, L_{z}$ and $Q$, it is then straightforward [23] to calculate $d p / d t, d e / d t$, and $d \theta_{\text {inc }} / d t$ (or $\left.d \iota / d t\right)$.

We should note that although perfectly well-behaved for all bound stable geodesics, the adiabatic approximation breaks down in a small region of the orbital parameters space very close to the separatrix, where the transition from an inspiral to a plunging orbit takes place [27]. However, since this region is expected to be very small ${ }^{2}$ and its impact on LISA waveforms rather hard to detect [27], we expect our results to be at least qualitatively correct also in this region of the space of parameters.

Accurate calculation of $d E / d t$ and $d L_{z} / d t$ in the adiabatic approximation involves solving the Teukolsky and Sasaki-Nakamura equations $[28,29]$. For generic orbits this has been done for the first time in Ref. [21]. The calculation of $d Q / d t$ for generic orbits is more involved. A formula for $d Q / d t$ has been recently derived [22], but has not yet been implemented (at least in a code to which we have access).

On the other hand, it is well-known that a circular orbit will remain circular under radiation reaction [30-32]. This constraint means that Teukolsky-based fluxes for $E$ and $L_{z}$ are sufficient to compute $d Q / d t$. Considering this limit, the rate of change $d Q / d t$ can be expressed in terms of $d E / d t$ and $d L / d t$ as

$$
\left(\frac{d Q}{d t}\right)_{\mathrm{circ}}=-\frac{N_{1}(p, \iota)}{N_{5}(p, \iota)}\left(\frac{d E}{d t}\right)_{\mathrm{circ}}-\frac{N_{4}(p, \iota)}{N_{5}(p, \iota)}\left(\frac{d L_{z}}{d t}\right)_{\mathrm{circ}},
$$

where

$$
\begin{gathered}
N_{1}(p, \iota) \equiv E(p, \iota) p^{4}+a^{2} E(p, \iota) p^{2} \\
-2 a M\left(L_{z}(p, \iota)-a E(p, \iota)\right) p, \\
N_{4}(p, \iota) \equiv\left(2 M p-p^{2}\right) L_{z}(p, \iota)-2 M a E(p, \iota) p, \\
N_{5}(p, \iota) \equiv\left(2 M p-p^{2}-a^{2}\right) / 2 .
\end{gathered}
$$

(These quantities are for a circular orbit of radius $p$.) Using this, it is simple to compute $d \theta_{\text {inc }} / d t$ (or $d \iota / d t$ ).

\footnotetext{
${ }^{2}$ Its width in $p / M$ is expected to be of the order of $\Delta p / M \sim$ $(\mu / M)^{2 / 5}$, where $\mu$ is the mass of the infalling body [27].
}

This procedure was followed in Ref. [14], using the code presented in Ref. [17], to determine the evolution of $\iota$; this analysis indicated that $d \iota / d t<0$ for circular nearly horizon-skimming orbits. As a first step to our more general analysis, we have repeated this calculation but using the improved Sasaki-Nakamura-Teukolsky code presented in Ref. [21]; we focused on the case $a=0.998 M$.

Rather to our surprise, we discovered that the fluxes $d E / d t$ and $d L_{z} / d t$ computed with this more accurate code indicate that $d \iota / d t>0$ (and $d \theta_{\text {inc }} / d t>0$ ) for all circular nearly horizon-skimming orbits - in stark contrast with what was found in Ref. [14]. As mentioned in the introduction, the rate of change of inclination angle appears as the difference of two quantities. These quantities nearly cancel (and indeed cancel exactly in the limit $a=$ 0 ); as such, small relative errors in their values can lead to large relative error in the inferred inclination evolution. Values for $d E / d t, d L_{z} / d t, d \iota / d t$, and $d \theta_{\text {inc }} / d t$ computed using the present code are shown in Table I in the columns with the header "Teukolsky."

\section{EVOLUTION OF $\theta_{\text {inc }}$ : NONCIRCULAR ORBITS}

The corrected behavior of circular nearly horizonskimming orbits has naturally led us to investigate the evolution of noncircular nearly horizon-skimming orbits. Since our code cannot be used to compute $d Q / d t$, we have resorted to a kludge approach, based on those described in Refs. [23,24]. In particular, we mostly follow the procedure developed by Gair and Glampedakis [24], though (as described below) importantly modified.

The basic idea of the kludge procedure is to use the functional form of $2 \mathrm{PN}$ fluxes $E, L_{z}$ and $Q$, but to correct the circular part of these fluxes using fits to circular Teukolsky data. As developed in Ref. [24], the fluxes are written

$$
\begin{aligned}
\left(\frac{d E}{d t}\right)_{\mathrm{GG}}= & \left(1-e^{2}\right)^{3 / 2}\left[\left(1-e^{2}\right)^{-3 / 2}\left(\frac{d E}{d t}\right)_{2 \mathrm{PN}}(p, e, \iota)\right. \\
& \left.-\left(\frac{d E}{d t}\right)_{2 \mathrm{PN}}(p, 0, \iota)+\left(\frac{d E}{d t}\right)_{\mathrm{fit} \operatorname{circ}}(p, \iota)\right], \\
\left(\frac{d L_{z}}{d t}\right)_{\mathrm{GG}}= & \left(1-e^{2}\right)^{3 / 2}\left[\left(1-e^{2}\right)^{-3 / 2}\left(\frac{d L_{z}}{d t}\right)_{2 \mathrm{PN}}(p, e, \iota)\right. \\
& \left.-\left(\frac{d L_{z}}{d t}\right)_{2 \mathrm{PN}}(p, 0, \iota)+\left(\frac{d L_{z}}{d t}\right)_{\mathrm{fitcirc}}(p, \iota)\right], \\
\left(\frac{d Q}{d t}\right)= & \left(1-e^{2}\right)^{3 / 2} \sqrt{Q(p, e, \iota)} \\
\times & {\left[\left(1-e^{2}\right)^{-3 / 2}\left(\frac{d Q / d t}{\sqrt{Q}}\right)_{2 \mathrm{PN}}(p, e, \iota)\right.} \\
- & \left.\left(\frac{d Q / d t}{\sqrt{Q}}\right)_{2 \mathrm{PN}}(p, 0, \iota)+\left(\frac{d Q / d t}{\sqrt{Q}}\right)_{\mathrm{fit} \operatorname{circ}}(p, \iota)\right] .
\end{aligned}
$$


TABLE I. Teukolsky-based fluxes and kludge fluxes [computed using Eqs. (40), (43), and (44)] for circular orbits about a hole with $a=0.998 M$; $\mu$ represents the mass of the infalling body. The Teukolsky-based fluxes have an accuracy of $10^{-6}$

\begin{tabular}{|c|c|c|c|c|c|c|c|c|c|c|c|}
\hline$\frac{p}{M}$ & $e$ & $\begin{array}{c}\theta_{\text {inc }} \\
\text { (deg.) }\end{array}$ & $\begin{array}{c}\iota \\
\text { (deg.) }\end{array}$ & $\begin{array}{l}\frac{d E}{d t} \times \frac{M^{2}}{\mu^{2}} \\
\text { (kludge) }\end{array}$ & $\begin{array}{c}\frac{d E}{d t} \times \frac{M^{2}}{\mu^{2}} \\
\text { (Teukolsky) }\end{array}$ & $\begin{array}{l}\frac{d L_{z}}{d t} \times \frac{M}{\mu^{2}} \\
\text { (kludge) }\end{array}$ & $\begin{array}{c}\frac{d L_{z}}{d t} \times \frac{M}{\mu^{2}} \\
\text { (Teukolsky) }\end{array}$ & $\begin{array}{l}\frac{d \iota}{d t} \times \frac{M}{\mu^{2}} \\
\text { (kludge) }\end{array}$ & $\begin{array}{c}\frac{d \iota}{d t} \times \frac{M}{\mu^{2}} \\
\text { (Teukolsky) }\end{array}$ & $\begin{array}{l}\frac{d \theta_{\text {inc }}}{d t} \times \frac{M}{\mu^{2}} \\
\text { (kludge) }\end{array}$ & $\begin{array}{c}\frac{d \theta_{\text {inc }}}{d t} \times \frac{M}{\mu^{2}} \\
(\text { Teukolsky) }\end{array}$ \\
\hline 1.3 & 0 & 0 & 0 & $9.108 \times 10^{-2}$ & $-9.109 \times 10^{-2}$ & $-2.258 \times 10^{-1}$ & $-2.259 \times 10^{-1}$ & 0 & 0 & 0 & 0 \\
\hline 1.3 & 0 & 10.4870 & 1.6773 & $9.328 \times 10^{-2}$ & $-9.332 \times 10^{-2}$ & $-2.304 \times 10^{-1}$ & $-2.306 \times 10^{-1}$ & $1.837 \times 10^{-2}$ & $1.839 \times 10^{-2}$ & $6.462 \times 10^{-3}$ & $6.475 \times 10^{-3}$ \\
\hline 1.3 & 0 & 14.6406 & 6.1303 & $9.588 \times 10^{-2}$ & $-9.588 \times 10^{-2}$ & $-2.359 \times 10^{-1}$ & $-2.360 \times 10^{-1}$ & $2.397 \times 10^{-2}$ & $2.400 \times 10^{-2}$ & $8.645 \times 10^{-3}$ & $8.667 \times 10^{-3}$ \\
\hline 1.3 & 0 & 17.7000 & 19.3172 & $-9.875 \times 10^{-2}$ & $-9.876 \times 10^{-2}$ & $-2.420 \times 10^{-1}$ & $-2.421 \times 10^{-1}$ & $2.728 \times 10^{-2}$ & $2.731 \times 10^{-2}$ & $1.007 \times 10^{-2}$ & $1.010 \times 10^{-2}$ \\
\hline 1.3 & 0 & 20.1636 & 21.8210 & $-1.019 \times 10^{-1}$ & $-1.019 \times 10^{-1}$ & $-2.486 \times 10^{-1}$ & $-2.488 \times 10^{-1}$ & $2.943 \times 10^{-2}$ & $2.950 \times 10^{-2}$ & $1.111 \times 10^{-2}$ & $1.117 \times 10^{-2}$ \\
\hline 1.4 & 0 & 0 & 0 & $-8.700 \times 10^{-2}$ & $-8.709 \times 10^{-2}$ & $-2.311 \times 10^{-1}$ & $-2.312 \times 10^{-1}$ & 0 & 0 & 0 & 0 \\
\hline 1.4 & 0 & 14.5992 & 16.0005 & $9.062 \times 10^{-2}$ & $-9.070 \times 10^{-2}$ & $-2.386 \times 10^{-1}$ & $-2.386 \times 10^{-1}$ & $2.316 \times 10^{-2}$ & $2.319 \times 10^{-2}$ & $8.823 \times 10^{-2} 3$ & $8.848 \times 10^{-3}$ \\
\hline 1.4 & 0 & 20.1756 & 21.7815 & $-9.520 \times 10^{-2}$ & $-9.526 \times 10^{-2}$ & $-2.482 \times 10^{-1}$ & $-2.482 \times 10^{-1}$ & $2.875 \times 10^{-2}$ & $2.877 \times 10^{-2}$ & $1.141 \times 10^{-2}$ & $1.143 \times 10^{-2}$ \\
\hline 1.4 & 0 & 24.1503 & 25.7517 & $-1.006 \times 10^{-1}$ & $-1.007 \times 10^{-1}$ & $-2.595 \times 10^{-1}$ & $-2.596 \times 10^{-1}$ & $3.140 \times 10^{-2}$ & $3.141 \times 10^{-2}$ & $1.289 \times 10^{-2}$ & $1.288 \times 10^{-2}$ \\
\hline 1.4 & 0 & 27.2489 & 04 & $1.067 \times 10^{-1}$ & $-1.068 \times 10^{-1}$ & $-2.725 \times 10^{-1}$ & $-2.725 \times 10^{-1}$ & $3.274 \times 10^{-2}$ & $3.275 \times 10^{-2}$ & $1.378 \times 10^{-2}$ & $1.377 \times 10^{-2}$ \\
\hline 1.5 & 0 & 0 & 0 & $-8.009 \times 10^{-2}$ & $-7.989 \times 10^{-2}$ & $-2.270 \times 10^{-1}$ & $-2.265 \times 10^{-1}$ & 0 & 0 & 0 & 0 \\
\hline 1.5 & 0 & 16.7836 & 18.1857 & $-8.401 \times 10^{-2}$ & $-8.383 \times 10^{-2}$ & $-2.348 \times 10^{-1}$ & $-2.343 \times 10^{-1}$ & $2.360 \times 10^{-2}$ & $2.351 \times 10^{-2}$ & $9.602 \times 10^{-3}$ & $9.545 \times 10^{-3}$ \\
\hline 1.5 & 0 & 23.0755 & 24.6167 & $-8.917 \times 10^{-2}$ & $-8.897 \times 10^{-2}$ & $-2.454 \times 10^{-1}$ & $-2.449 \times 10^{-1}$ & $2.872 \times 10^{-2}$ & $2.863 \times 10^{-2}$ & $1.228 \times 10^{-2}$ & $1.222 \times 10^{-2}$ \\
\hline 1.5 & 0 & 27.4892 & 28.9670 & $-9.537 \times 10^{-2}$ & $-9.516 \times 10^{-2}$ & $-2.583 \times 10^{-1}$ & $-2.579 \times 10^{-1}$ & $3.091 \times 10^{-2}$ & $3.082 \times 10^{-2}$ & $1.372 \times 10^{-2}$ & $1.367 \times 10^{-2}$ \\
\hline 1.5 & 0 & 30.8795 & 32.2231 & $-1.025 \times 10^{-1}$ & $-1.023 \times 10^{-1}$ & $-2.733 \times 10^{-1}$ & $-2.728 \times 10^{-1}$ & $3.184 \times 10^{-2}$ & $3.173 \times 10^{-2}$ & $1.452 \times 10^{-2}$ & $1.443 \times 10^{-2}$ \\
\hline 1.6 & 0 & 0 & 0 & $-7.181 \times 10^{-2}$ & $-7.156 \times 10^{-2}$ & $-2.168 \times 10^{-1}$ & $-2.162 \times 10^{-1}$ & 0 & 0 & 0 & 0 \\
\hline 1.6 & 0 & 18.3669 & 19.7220 & $-7.568 \times 10^{-2}$ & $-7.545 \times 10^{-2}$ & $-2.242 \times 10^{-1}$ & $-2.237 \times 10^{-1}$ & $2.240 \times 10^{-2}$ & $2.229 \times 10^{-2}$ & $9.600 \times 10^{-3}$ & $9.515 \times 10^{-3}$ \\
\hline 1.6 & 0 & 25.1720 & 26.6245 & $-8.084 \times 10^{-2}$ & $-8.062 \times 10^{-2}$ & $-2.346 \times 10^{-1}$ & $-2.341 \times 10^{-1}$ & $2.701 \times 10^{-2}$ & $2.685 \times 10^{-2}$ & $1.223 \times 10^{-2}$ & $1.210 \times 10^{-2}$ \\
\hline 1.6 & 0 & 29.9014 & 120 & $-8.708 \times 10^{-2}$ & $-8.687 \times 10^{-2}$ & $-2.474 \times 10^{-1}$ & $-2.470 \times 10^{-1}$ & $2.889 \times 10^{-2}$ & $2.872 \times 10^{-2}$ & $1.363 \times 10^{-2}$ & $1.349 \times 10^{-2}$ \\
\hline 1.6 & 0 & 33.5053 & 34.7 & $-9.425 \times 10^{-2}$ & $-9.399 \times 10^{-2}$ & $-2.622 \times 10^{-1}$ & $-2.616 \times 10^{-1}$ & $2.964 \times 10^{-2}$ & $2.951 \times 10^{-2}$ & $1.441 \times 10^{-2}$ & $1.432 \times 10^{-2}$ \\
\hline 1.7 & 0 & 0 & 0 & $-6.332 \times 10^{-2}$ & $-6.317 \times 10^{-2}$ & $-2.034 \times 10^{-1}$ & $-2.031 \times 10^{-1}$ & 0 & 0 & 0 & 0 \\
\hline 1.7 & 0 & 19.6910 & 20 & $-6.702 \times 10^{-2}$ & $-6.687 \times 10^{-2}$ & $-2.101 \times 10^{-1}$ & $-2.098 \times 10^{-1}$ & $2.057 \times 10^{-2}$ & $2.052 \times 10^{-2}$ & $9.202 \times 10^{-3}$ & $9.171 \times 10^{-3}$ \\
\hline 1.7 & 0 & 26.9 & 28 & -7.1 & $-7.184 \times 10^{-2}$ & $-2.199 \times 10^{-1}$ & $-2.196 \times 1$ & $2.467 \times 1$ & $2.456 \times 10^{-2}$ & $1.170 \times 1$ & $1.162 \times 10^{-2}$ \\
\hline 1.7 & 0 & 31.9218 & 33.1786 & $-7.794 \times 10^{-2}$ & $-7.782 \times 10^{-2}$ & $-2.319 \times 10^{-1}$ & $-2.316 \times 10^{-1}$ & $2.632 \times 10^{-2}$ & $2.620 \times 10^{-2}$ & $1.306 \times 10^{-2}$ & $1.296 \times 10^{-2}$ \\
\hline 1.7 & 0 & 35.7100 & 36.8118 & $-8.475 \times 10^{-2}$ & $-8.465 \times 10^{-2}$ & $-2.457 \times 10^{-1}$ & $-2.455 \times 10^{-1}$ & $2.698 \times 10^{-2}$ & $2.686 \times 10^{-2}$ & $1.384 \times 10^{-2}$ & $1.373 \times 10^{-2}$ \\
\hline 1.8 & 0 & 0 & 0 & -5.5 & $-5.528 \times 10^{-2}$ & $-1.888 \times 10^{-1}$ & $-1.887 \times 10^{-1}$ & 0 & 0 & 0 & 0 \\
\hline 1.8 & 0 & 20.8804 & 22.1128 & $-5.879 \times 10^{-2}$ & $-5.874 \times 10^{-2}$ & $-1.948 \times 10^{-1}$ & $-1.946 \times 10^{-1}$ & $1.858 \times 10^{-2}$ & $1.858 \times 10^{-2}$ & $8.635 \times 10^{-3}$ & $8.639 \times 10^{-3}$ \\
\hline 1.8 & 0 & 28.5007 & 9.7791 & $-6.343 \times 10^{-2}$ & $-6.336 \times 10^{-2}$ & $-2.036 \times 10^{-1}$ & $-2.035 \times 10^{-1}$ & $2.221 \times 10^{-2}$ & $2.223 \times 10^{-2}$ & $1.098 \times 10^{-2}$ & $1.101 \times 10^{-2}$ \\
\hline 1.8 & 0 & 33.7400 & 34.9034 & $-6.901 \times 10^{-2}$ & $-6.894 \times 10^{-2}$ & $-2.146 \times 10^{-1}$ & $-2.144 \times 10^{-1}$ & $2.368 \times 10^{-2}$ & $2.371 \times 10^{-2}$ & $1.228 \times 10^{-2}$ & $1.232 \times 10^{-2}$ \\
\hline 1.8 & 0 & 37.6985 & 38.7065 & $-7.533 \times 10^{-2}$ & $-7.533 \times 10^{-2}$ & $-2.271 \times 10^{-1}$ & $-2.271 \times 10^{-1}$ & $2.429 \times 10^{-2}$ & $2.427 \times 10^{-2}$ & $1.306 \times 10^{-2}$ & $1.303 \times 10^{-2}$ \\
\hline 1.9 & 0 & 0 & 0 & $-4.809 \times 10^{-2}$ & $-4.811 \times 10^{-2}$ & $-1.740 \times 10^{-1}$ & $-1.740 \times 10^{-1}$ & 0 & 0 & 0 & 0 \\
\hline 1.9 & 0 & 21.9900 & 23.1615 & $-5.132 \times 10^{-2}$ & $-5.134 \times 10^{-2}$ & $-1.792 \times 10^{-1}$ & $-1.793 \times 10^{-1}$ & $1.666 \times 10^{-2}$ & $1.664 \times 10^{-2}$ & $8.022 \times 1$ & $8.007 \times 10^{-3}$ \\
\hline 1.9 & 0 & 29.9708 & 31.1702 & $-5.562 \times 10^{-2}$ & $-5.564 \times 10^{-2}$ & $-1.872 \times 10^{-1}$ & $-1.872 \times 10^{-1}$ & $1.986 \times 10^{-2}$ & $1.987 \times 10^{-2}$ & $1.019 \times 10^{-2}$ & $1.020 \times 10^{-2}$ \\
\hline 1.9 & 0 & 35.4385 & 36.5176 & $-6.078 \times 10^{-2}$ & $-6.077 \times 10^{-2}$ & $-1.971 \times 10^{-1}$ & $-1.970 \times 10^{-1}$ & $2.118 \times 10^{-2}$ & $2.122 \times 10^{-2}$ & $1.143 \times 10^{-2}$ & $1.148 \times 10^{-2}$ \\
\hline 1.9 & 0 & 39.5592 & 40.4847 & $-6.659 \times 10^{-2}$ & $-6.658 \times 10^{-2}$ & $-2.082 \times 10^{-1}$ & $-2.082 \times 10^{-1}$ & $2.177 \times 10^{-2}$ & $2.182 \times 10^{-2}$ & $1.222 \times 10^{-2}$ & $1.228 \times 10^{-2}$ \\
\hline 2.0 & 0 & 0 & 0 & $-4.174 \times 10^{-2}$ & $-4.175 \times 10^{-2}$ & $-1.598 \times 10^{-1}$ & $-1.598 \times 10^{-1}$ & 0 & 0 & 0 & 0 \\
\hline 2.0 & 0 & 23.0471 & 24.1605 & $-4.471 \times 10^{-2}$ & $-4.472 \times 10^{-2}$ & $-1.643 \times 10^{-1}$ & $-1.643 \times 10^{-1}$ & $1.489 \times 10^{-2}$ & $1.489 \times 10^{-2}$ & $7.425 \times 10^{-3}$ & $7.424 \times 10^{-3}$ \\
\hline 2.0 & 0 & 31.3715 & 32.4978 & $-4.867 \times 10^{-2}$ & $-4.871 \times 10^{-2}$ & $-1.713 \times 10^{-1}$ & $-1.714 \times 10^{-1}$ & $1.773 \times 10^{-2}$ & $1.770 \times 10^{-2}$ & $9.436 \times 10^{-3}$ & $9.411 \times 10^{-3}$ \\
\hline 2.0 & 0 & 37.0583 & 38.0608 & $-5.341 \times 10^{-2}$ & $-5.345 \times 10^{-2}$ & $-1.801 \times 10^{-1}$ & $-1.801 \times 10^{-1}$ & $1.893 \times 10^{-2}$ & $1.889 \times 10^{-2}$ & $1.062 \times 10^{-2}$ & $1.057 \times 10^{-2}$ \\
\hline 2.0 & 0 & 41.3358 & 42.1876 & $-5.873 \times 10^{-2}$ & $-5.875 \times 10^{-2}$ & $-1.900 \times 10^{-1}$ & $-1.900 \times 10^{-1}$ & $1.950 \times 10^{-2}$ & $1.948 \times 10^{-2}$ & $1.141 \times 10^{-2}$ & $1.138 \times 10^{-2}$ \\
\hline
\end{tabular}


TABLE II. As in Table I but for noncircular orbits; the Teukolsky-based fluxes for $E$ and $L_{z}$ have an accuracy of $10^{-3}$. Note that our code, as all the Teukolsky-based code that we are aware of, presently does not have the capability to compute inclination angle evolution for generic orbits.

\begin{tabular}{|c|c|c|c|c|c|c|c|c|c|}
\hline$\frac{p}{M}$ & $e$ & $\begin{array}{c}\theta_{\text {inc }} \\
\text { (deg.) }\end{array}$ & $\begin{array}{c}\iota \\
\text { (deg.) }\end{array}$ & $\begin{array}{l}\frac{d E}{d t} \times \frac{M^{2}}{\mu^{2}} \\
\text { (kludge) }\end{array}$ & $\begin{array}{c}\frac{d E}{d t} \times \frac{M^{2}}{\mu^{2}} \\
\text { (Teukolsky) }\end{array}$ & $\begin{array}{l}\frac{d L_{z}}{d t} \times \frac{M}{\mu^{2}} \\
\text { (kludge) }\end{array}$ & $\begin{array}{c}\frac{d L_{z}}{d t} \times \frac{M}{\mu^{2}} \\
\text { (Teukolsky) }\end{array}$ & $\begin{array}{l}\frac{d \iota}{d t} \times \frac{M}{\mu^{2}} \\
\text { (kludge) }\end{array}$ & $\begin{array}{l}\frac{d \theta_{\text {inc }}}{d t} \times \frac{M}{\mu^{2}} \\
\text { (kludge) }\end{array}$ \\
\hline 1.3 & & 0 & 0 & 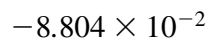 & $8.804 \times 10$ & $2.098 \times 10^{-1}$ & $-2.098 \times 10$ & 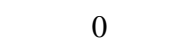 & U \\
\hline 1.4 & & 0 & 0 & & $8.719 \times 1$ & $2.274 \times$ & $-2.275 \times 10^{-1}$ & 0 & 0 \\
\hline 1.4 & 1 & 8 & & $.110 \times 10^{-2}$ & $-8.736 \times 10^{-2}$ & $-2.355 \times 10^{-1}$ & $-2.273 \times 10^{-1}$ & $4.066 \times 10^{-2}$ & $2.938 \times 10^{-2}$ \\
\hline 1.4 & 0.1 & 16 & & $1.030 \times 10^{-1}$ & $-8.958 \times 1$ & $2.602 \times 1$ & $-2.309 \times 10^{-1}$ & $428 \times 10^{-2}$ & $5.475 \times 10^{-2}$ \\
\hline 1.4 & .1 & 24 & & $1.243 \times 1$ & $-9.771 \times 10$ & $-3.037 \times 10$ & $-2.415 \times 10^{-1}$ & $.663 \times 10^{-2}$ & $7.316 \times 10^{-2}$ \\
\hline 1.5 & 0.1 & 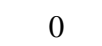 & 0 & $8.069 \times 10^{-2}$ & $-8.095 \times 10^{-2}$ & $-2.255 \times 10^{-1}$ & $-2.260 \times 10^{-1}$ & 0 & 0 \\
\hline 1.5 & .1 & 8 & & $8.323 \times 10$ & $-8.133 \times 10$ & $-2.310 \times 10$ & $-2.264 \times 10^{-1}$ & $2.996 \times 1$ & $2.070 \times 10^{-2}$ \\
\hline 1.5 & 0.1 & 16 & 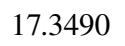 & $9.121 \times 10^{-2}$ & $-8.395 \times 10$ & $-2.483 \times 10^{-1}$ & $-2.314 \times 10^{-1}$ & $5.512 \times 10^{-2}$ & $3.888 \times 10^{-2}$ \\
\hline 1.5 & 0.1 & 24 & & 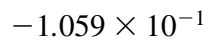 & $-8.980 \times 10$ & $-2.792 \times 1$ & $-2.423 \times 10^{-1}$ & $7.255 \times 1$ & $5.264 \times 10^{-2}$ \\
\hline 1.6 & 0.1 & 0 & 0 & 7.255 & $-7.281 \times 1$ & $-2.161 \times 1$ & $-2.168 \times 10^{-1}$ & 0 & 0 \\
\hline 1.6 & 0.1 & $\delta$ & & $7.430 \times 10$ & $-7.321 \times 10^{-2}$ & $-2.201 \times 10^{-1}$ & $-2.173 \times 10^{-1}$ & $2.258 \times 1$ & $1.502 \times 10^{-2}$ \\
\hline 1.6 & 0.1 & 16 & & $7.986 \times 1$ & $-7.533 \times 10$ & $-2.323 \times 1$ & $-2.212 \times 1$ & $.179 \times$ & $2.839 \times 10^{-2}$ \\
\hline 1.6 & 0.1 & 2 & 8 & $-9.025 \times 1$ & $-8.040 \times 10$ & $-2.547 \times 1$ & $-2.309 \times 10^{-1}$ & $5.554 \times 1$ & $3.886 \times 10^{-2}$ \\
\hline 1.6 & 0.1 & 32 & 3 & $-1.082 \times 10^{-1}$ & $-9.435 \times 10$ & $-2.920 \times 10^{-1}$ & $-2.551 \times 1$ & $6.316 \times 1$ & $4.559 \times 10^{-2}$ \\
\hline 1.7 & 0.1 & 0 & 0 & 5.427 & $-6.440 \times 1$ & $-2.036 \times 1$ & $-2.040 \times 10^{-1}$ & 0 & 0 \\
\hline 1.7 & 0.1 & 8 & & $6.552 \times 1$ & $-6.478 \times 10^{-2}$ & $-2.065 \times 10^{-1}$ & $-2.045 \times 10^{-1}$ & $1.742 \times 1$ & $1.124 \times 10^{-2}$ \\
\hline 1.7 & 0.1 & 1 & & & & $-2.154 \times$ & -2.0 & $240 \times$ & \\
\hline 1.7 & 0.1 & 2 & & $-7.707 \times 10^{-2}$ & $-7.052 \times 10^{-2}$ & $-2.317 \times 10^{-1}$ & $-2.150 \times 10^{-1}$ & $4.342 \times 10^{-2}$ & $2.948 \times 10^{-2}$ \\
\hline 1.7 & 0.1 & 32 & 6 & $-9.009 \times 10^{-2}$ & $-7.959 \times 10^{-2}$ & $-2.590 \times 10^{-1}$ & $-2.324 \times 10^{-1}$ & $4.998 \times 10^{-2}$ & $3.512 \times 10^{-2}$ \\
\hline 1.8 & 0. & 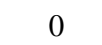 & 0 & 640 & $-5.640 \times 1$ & $-1.897 \times 1$ & $-1.897 \times 10^{-1}$ & 0 & 0 \\
\hline 1.8 & 0.1 & 8 & & $-5.732 \times 10^{-2}$ & $-5.676 \times 10^{-2}$ & $-1.918 \times 10^{-1}$ & $-1.902 \times 10^{-1}$ & $1.371 \times 1$ & $8.640 \times 10^{-3}$ \\
\hline 1.8 & 0.1 & 1 & & & & -1.9 & -1.9 & -2 & $47 \times 10^{-2}$ \\
\hline 1.8 & 0.1 & $2-3+2$ & & $-6.588 \times 10^{-2}$ & $-6.139 \times 10^{-2}$ & $-2.105 \times 10^{-1}$ & $-1.983 \times 10^{-1}$ & $3.456 \times 10^{-2}$ & $2.291 \times 10^{-2}$ \\
\hline 1.8 & 0.1 & 32 & 33.2 & $-7.555 \times 10^{-2}$ & $-6.849 \times 10^{-2}$ & $-2.307 \times 10^{-1}$ & $-2.120 \times 10^{-1}$ & $4.020 \times 10^{-2}$ & $2.765 \times 10^{-2}$ \\
\hline 1.9 & 0. & 0 & 0 & $-4.915 \times 10^{-2}$ & $-4.911 \times 10^{-2}$ & $-1.753 \times 10^{-1}$ & $-1.751 \times 10^{-1}$ & 0 & 0 \\
\hline 1.9 & 0.1 & 8 & & $-4.985 \times 10^{-2}$ & $-4.945 \times 10^{-2}$ & $-1.768 \times 10^{-1}$ & $-1.755 \times 10^{-1}$ & $1.097 \times 1$ & $6.791 \times 10^{-3}$ \\
\hline 1.9 & 0.1 & 1 & & -5.2 & -5 & $-1.817 \times 1$ & $-1.774 \times 1$ & -2 & $1.298 \times 10^{-2}$ \\
\hline 1.9 & 0.1 & 2 & & $-5.633 \times 10^{-2}$ & $-5.328 \times 10^{-2}$ & $-1.908 \times 10^{-1}$ & $-1.819 \times 10^{-1}$ & $2.788 \times 10^{-2}$ & $1.816 \times 10^{-2}$ \\
\hline 1.9 & 0.1 & 32 & 33.15 & $-6.364 \times 10^{-2}$ & $-5.870 \times 10^{-2}$ & $-2.059 \times 10^{-1}$ & $-1.920 \times 10^{-1}$ & $3.272 \times 10^{-2}$ & $2.214 \times 10^{-2}$ \\
\hline 2.0 & 0.1 & 0 & 0 & $-4.263 \times 10^{-2}$ & $-4.264 \times 10^{-2}$ & $-1.607 \times 10^{-1}$ & $-1.608 \times 10^{-1}$ & 0 & 0 \\
\hline 2.0 & 0.1 & 8 & & $-4.316 \times 10^{-2}$ & $-4.292 \times 10^{-2}$ & $-1.619 \times 10^{-1}$ & $-1.611 \times 10^{-1}$ & $8.862 \times 1$ & $5.424 \times 10^{-3}$ \\
\hline 2.0 & 0.1 & 16 & & $-4.488 \times 10^{-2}$ & $-4.390 \times 10^{-2}$ & $-1.656 \times 10^{-1}$ & $-1.625 \times 10^{-1}$ & $1.666 \times 10^{-2}$ & $1.039 \times 10^{-2}$ \\
\hline 2.0 & 0.1 & 24 & & $-4.815 \times 10^{-2}$ & $-4.604 \times 10^{-2}$ & $-1.724 \times 10^{-1}$ & $-1.660 \times 10^{-1}$ & $2.271 \times 10^{-2}$ & $1.459 \times 10^{-2}$ \\
\hline 2.0 & 0.1 & 32 & & $-5.376 \times 10^{-2}$ & $-5.031 \times 10^{-2}$ & $-1.838 \times 10^{-1}$ & $-1.736 \times 10^{-1}$ & $2.684 \times 10^{-2}$ & $1.793 \times 10^{-2}$ \\
\hline 2.0 & 0.1 & 40 & 40.8954 & $-6.339 \times 10^{-2}$ & $-6.236 \times 10^{-2}$ & $-2.027 \times 10^{-1}$ & $-1.967 \times 10^{-1}$ & $2.917 \times 10^{-2}$ & $2.036 \times 10^{-2}$ \\
\hline
\end{tabular}

The post-Newtonian fluxes $(d E / d t)_{2 \mathrm{PN}},\left(d L_{z} / d t\right)_{2 \mathrm{PN}}$ and $(d Q / d t)_{2 \mathrm{PN}}$ are given in the Appendix [particularly Eqs. (A1)-(A3)].

Since for circular orbits the fluxes $d E / d t, d L_{z} / d t$ and $d Q / d t$ are related through Eq. (34), only two fits to circular Teukolsky data are needed. One possible choice is to fit $d L_{z} / d t$ and $d \iota / d t$, and then use the circularity constraint to obtain $^{3}[24]$

\footnotetext{
${ }^{3}$ This choice might seem more involved than fitting directly $d L_{z} / d t$ and $d Q / d t$, but, as noted by Gair and Glampedakis, ensures more sensible results for the evolution of the inclination angle. This generates more physically realistic inspirals [24].
}

$$
\begin{aligned}
& \left(\frac{d Q / d t}{\sqrt{Q}}\right)_{\text {fit circ }}(p, \iota)=2 \tan \iota\left[\left(\frac{d L_{z}}{d t}\right)_{\text {fit circ }}+\frac{\sqrt{Q(p, 0, \iota)}}{\sin ^{2} \iota}\right. \\
& \left.\times\left(\frac{d \iota}{d t}\right)_{\text {fit circ }}\right] \text {, } \\
& \left(\frac{d E}{d t}\right)_{\text {fit circ }}(p, \iota)=-\frac{N_{4}(p, \iota)}{N_{1}(p, \iota)}\left(\frac{d L z}{d t}\right)_{\text {fit circ }}(p, \iota)-\frac{N_{5}(p, \iota)}{N_{1}(p, \iota)} \\
& \times \sqrt{Q(p, 0, \iota)}\left(\frac{d Q / d t}{\sqrt{Q}}\right)_{\text {fit circ }}(p, \iota) .
\end{aligned}
$$

As stressed in Ref. [24], one does not expect these fluxes to work well in the strong field, both because the postNewtonian approximation breaks down close to the black 
TABLE III. As in Table II, but for additional values of eccentricity $e$; the Teukolsky-based fluxes for $E$ and $L_{z}$ have an accuracy of $10^{-3}$.

\begin{tabular}{|c|c|c|c|c|c|c|c|c|c|}
\hline$\frac{p}{M}$ & $e$ & $\begin{array}{c}\theta_{\text {inc }} \\
\text { (deg.) }\end{array}$ & $\begin{array}{c}\iota \\
\text { (deg.) }\end{array}$ & $\begin{array}{l}\frac{d E}{d t} \times \frac{M^{2}}{\mu^{2}} \\
\text { (kludge) }\end{array}$ & $\begin{array}{c}\frac{d E}{d t} \times \frac{M^{2}}{\mu^{2}} \\
(\text { Teukolsky) }\end{array}$ & $\begin{array}{l}\frac{d L_{z}}{d t} \times \frac{M}{\mu^{2}} \\
\text { (kludge) }\end{array}$ & $\begin{array}{c}\frac{d L_{z}}{d t} \times \frac{M}{\mu^{2}} \\
\text { (Teukolsky) }\end{array}$ & $\begin{array}{l}\frac{d \iota}{d t} \times \frac{M}{\mu^{2}} \\
\text { (kludge) }\end{array}$ & $\begin{array}{l}\frac{d \theta_{\text {inc }}}{d t} \times \frac{M}{\mu^{2}} \\
\text { (kludge) }\end{array}$ \\
\hline 1.4 & 0.2 & 0 & 0 & $-8.636 \times 10^{-2}$ & $-8.642 \times 10^{-2}$ & $-2.119 \times 10^{-1}$ & $-2.121 \times 10^{-1}$ & 0 & 0 \\
\hline 1.4 & .2 & 8 & 8.8215 & $9.853 \times 10^{-2}$ & $-8.240 \times 10^{-2}$ & $-2.374 \times 10^{-1}$ & $-2.015 \times 10^{-1}$ & $1.148 \times 10^{-1}$ & $9.714 \times 10^{-2}$ \\
\hline 1.5 & 0.2 & 0 & 0 & $-8.362 \times 10^{-2}$ & $-8.349 \times 10^{-2}$ & $-2.236 \times 10^{-1}$ & $-2.230 \times 10^{-1}$ & 0 & 0 \\
\hline 1.5 & 0.2 & 8 & 8.7595 & $9.141 \times 10^{-2}$ & $-8.276 \times 10^{-2}$ & $-2.410 \times 10^{-1}$ & $-2.206 \times 10^{-1}$ & $7.893 \times 10^{-2}$ & $6.549 \times 10^{-2}$ \\
\hline 1.5 & 0.2 & 16 & 17.2957 & $-1.145 \times 10^{-1}$ & $-8.394 \times 10^{-2}$ & $-2.915 \times 10^{-1}$ & $-2.215 \times 10^{-1}$ & $1.466 \times 10^{-1}$ & $1.230 \times 10^{-1}$ \\
\hline 1.5 & 0.2 & 24 & 25.4608 & $-1.524 \times 10^{-1}$ & $-9.230 \times 10^{-2}$ & $-3.712 \times 10^{-1}$ & $-2.357 \times 10^{-1}$ & $1.952 \times 10^{-1}$ & $1.661 \times 10^{-1}$ \\
\hline 1.6 & 0.2 & 0 & 0 & $-7.596 \times 10^{-2}$ & $-7.616 \times 10^{-2}$ & $-2.171 \times 10^{-1}$ & $-2.176 \times 10^{-1}$ & 0 & 0 \\
\hline 1.6 & 0.2 & 8 & 8.6935 & $-8.111 \times 10^{-2}$ & $-7.641 \times 10^{-2}$ & $-2.292 \times 10^{-1}$ & $-2.177 \times 10^{-1}$ & $5.520 \times 10^{-2}$ & $4.502 \times 10^{-2}$ \\
\hline 1.6 & 0.2 & 16 & 17.1994 & $-9.649 \times 10^{-2}$ & $-7.798 \times 10^{-2}$ & $-2.647 \times 10^{-1}$ & $-2.198 \times 10^{-1}$ & $1.032 \times 10^{-1}$ & $8.500 \times 10^{-2}$ \\
\hline 1.6 & 0.2 & 24 & 25.3891 & $-1.221 \times 10^{-1}$ & $-8.314 \times 10^{-2}$ & $-3.212 \times 10^{-1}$ & $-2.288 \times 10^{-1}$ & $1.388 \times 10^{-1}$ & $1.160 \times 10^{-1}$ \\
\hline 1.7 & 0.2 & 0 & 0 & $-6.765 \times 10^{-2}$ & $-6.799 \times 10^{-2}$ & $-2.057 \times 10^{-1}$ & $-2.068 \times 10^{-1}$ & 0 & 0 \\
\hline 1.7 & 0.2 & 8 & 8.6329 & $-7.116 \times 10^{-2}$ & $-6.813 \times 10^{-2}$ & $-2.144 \times 10^{-1}$ & $-2.066 \times 10^{-1}$ & $3.963 \times 10^{-2}$ & $3.176 \times 10^{-2}$ \\
\hline 1.7 & 0.2 & 16 & 17.1064 & $-8.171 \times 10^{-2}$ & $-6.995 \times 10^{-2}$ & $-2.398 \times 10^{-1}$ & $-2.096 \times 10^{-1}$ & $7.441 \times 10^{-2}$ & $6.024 \times 10^{-2}$ \\
\hline 1.7 & 0.2 & 24 & 308 & $-9.948 \times 10^{-2}$ & $-7.443 \times 10^{-2}$ & $-2.806 \times 10^{-1}$ & $-2.178 \times 10^{-1}$ & $1.009 \times 1$ & $8.290 \times 10^{-2}$ \\
\hline 1.7 & 0.2 & 32 & .20 & $-1.257 \times 10^{-1}$ & $-8.558 \times 10^{-2}$ & $-3.371 \times 10^{-1}$ & $-2.366 \times 10^{-1}$ & $1.175 \times 10^{-1}$ & $9.806 \times 10^{-2}$ \\
\hline 1.8 & 0.2 & 0 & 0 & $-5.965 \times 10$ & $-5.962 \times 10^{-2}$ & $-1.927 \times 10^{-1}$ & $-1.926 \times 10^{-1}$ & 0 & 0 \\
\hline 1.8 & 0.2 & 8 & 789 & $-6.211 \times 10^{-2}$ & $-5.997 \times 10^{-2}$ & $-1.990 \times 1$ & $-1.930 \times 10^{-1}$ & $2.919 \times 10^{-2}$ & $2.300 \times 10^{-2}$ \\
\hline 1.8 & 0.2 & 16 & 702 & $-6.953 \times 10^{-2}$ & $-6.147 \times 10^{-2}$ & $-2.175 \times 10^{-1}$ & $-1.954 \times 10^{-1}$ & $5.504 \times 10^{-2}$ & $4.380 \times 10^{-2}$ \\
\hline 1.8 & 0.2 & 24 & & $-8.216 \times 1$ & $-6.502 \times 10^{-2}$ & $-2.474 \times 1$ & $-2.016 \times 1$ & $7.515 \times 1$ & $6.068 \times 10^{-2}$ \\
\hline 1.8 & 0.2 & 32 & 33.16 & $-1.009 \times 10^{-1}$ & $-7.410 \times 10^{-2}$ & $-2.890 \times 1$ & $-2.190 \times 10^{-1}$ & $8.839 \times 10^{-2}$ & $7.258 \times 10^{-2}$ \\
\hline 1.9 & 0.2 & 0 & 0 & $-5.218 \times 10^{-2}$ & $-5.210 \times 10^{-2}$ & $-1.786 \times 10^{-1}$ & $-1.783 \times 10^{-1}$ & 0 & 0 \\
\hline 1.9 & 0.2 & 8 & & & $-5.244 \times 1$ & $-1.833 \times 1$ & $-1.787 \times 1$ & $2.197 \times 1$ & $1.704 \times 10^{-2}$ \\
\hline 1.9 & 0.2 & 16 & 16.9441 & $-5.928 \times 10^{-2}$ & $-5.373 \times 10^{-2}$ & $-1.970 \times 10^{-1}$ & $-1.807 \times 10^{-1}$ & $4.156 \times 1$ & $3.254 \times 10^{-2}$ \\
\hline 1.9 & 0.2 & 24 & 1518 & $-6.843 \times 10^{-2}$ & $-5.669 \times 10^{-2}$ & $-2.192 \times 10^{-1}$ & $-1.858 \times 10^{-1}$ & $5.706 \times 10^{-2}$ & $4.535 \times 10^{-2}$ \\
\hline 1.9 & 0.2 & 32 & & & $-6.277 \times 10^{-2}$ & $-2.502 \times 1$ & $-1.966 \times 1$ & $6.767 \times 10^{-2}$ & $5.475 \times 10^{-2}$ \\
\hline 2.0 & 0.2 & 0 & 0 & $-4.528 \times 10^{-2}$ & $-4.530 \times 10^{-2}$ & $-1.637 \times 10^{-1}$ & $-1.638 \times 10^{-1}$ & 0 & 0 \\
\hline 2.0 & 0.2 & 8 & م & $-4.657 \times 10^{-2}$ & $-4.557 \times 10^{-2}$ & $-1.671 \times 10^{-1}$ & $-1.641 \times 10^{-1}$ & $1.679 \times 10^{-2}$ & $1.283 \times 10^{-2}$ \\
\hline 2.0 & 0.2 & 16 & & & $-4.664 \times 10^{-2}$ & $-1.774 \times 10^{-1}$ & $-1.657 \times 10^{-1}$ & $3.184 \times 10^{-2}$ & $2.457 \times 10^{-2}$ \\
\hline 2.0 & 0.2 & 24 & 0 & $-5.725 \times 10^{-2}$ & $-4.904 \times 10^{-2}$ & $-1.941 \times 10^{-1}$ & $-1.696 \times 10^{-1}$ & $4.391 \times 10^{-2}$ & $3.440 \times 10^{-2}$ \\
\hline 2.0 & 0.2 & 32 & 33.0730 & $-6.743 \times 10^{-2}$ & $-5.427 \times 10^{-2}$ & $-2.175 \times 10^{-1}$ & $-1.793 \times 10^{-1}$ & $5.243 \times 10^{-2}$ & $4.184 \times 10^{-2}$ \\
\hline 1.5 & 0.3 & 0 & 0 & $-8.481 \times 10^{-2}$ & $-8.478 \times 10^{-2}$ & $-2.094 \times 10^{-1}$ & $-2.094 \times 10^{-1}$ & 0 & 0 \\
\hline 1.5 & 0.3 & 8 & 8 & $-1.006 \times 10^{-1}$ & $-7.824 \times 10^{-2}$ & $-2.442 \times 10^{-1}$ & $-1.934 \times 10^{-1}$ & $1.484 \times 10^{-1}$ & $1.301 \times 10^{-1}$ \\
\hline 1.5 & 0.3 & 16 & 17.20 & $-1.469 \times 10^{-1}$ & $-7.811 \times 10^{-2}$ & $-3.435 \times 10^{-1}$ & $-1.864 \times 10^{-1}$ & $2.766 \times 10^{-1}$ & $2.440 \times 10^{-1}$ \\
\hline 1.6 & 0.3 & 0 & 0 & $-8.144 \times 10^{-2}$ & $-8.123 \times 10^{-2}$ & $-2.183 \times 10^{-1}$ & $-2.178 \times 10^{-1}$ & 0 & 0 \\
\hline 1.6 & 0.3 & 8 & 8.6498 & $-9.182 \times 10^{-2}$ & $-7.807 \times 10^{-2}$ & $-2.426 \times 10^{-1}$ & $-2.095 \times 10^{-1}$ & $1.028 \times 10^{-1}$ & $8.918 \times 10^{-2}$ \\
\hline 1.6 & 0.3 & 16 & 1246 & $-1.223 \times 10^{-1}$ & $-8.089 \times 10^{-2}$ & $-3.122 \times 10^{-1}$ & $-2.144 \times 10^{-1}$ & $1.928 \times 10^{-1}$ & $1.683 \times 10^{-1}$ \\
\hline 1.6 & 0.3 & 24 & 25.3046 & $-1.716 \times 10^{-1}$ & $-8.666 \times 10^{-2}$ & $-4.197 \times 10^{-1}$ & $-2.229 \times 10^{-1}$ & $2.607 \times 10^{-1}$ & $2.295 \times 10^{-1}$ \\
\hline 1.7 & 0.3 & 0 & 0 & $-7.362 \times 10^{-2}$ & $-7.314 \times 10^{-2}$ & $-2.104 \times 10^{-1}$ & $-2.095 \times 10^{-1}$ & 0 & 0 \\
\hline 1.7 & 0.3 & 8 & 8 & $-8.060 \times 10^{-2}$ & $-7.224 \times 10^{-2}$ & $-2.277 \times 10^{-1}$ & $-2.065 \times 10^{-1}$ & $7.240 \times 10^{-2}$ & $6.224 \times 10^{-2}$ \\
\hline 1.7 & 0.3 & 16 & .0415 & $-1.013 \times 10^{-1}$ & $-7.369 \times 10^{-2}$ & $-2.774 \times 10^{-1}$ & $-2.084 \times 10^{-1}$ & $1.365 \times 10^{-1}$ & $1.180 \times 10^{-1}$ \\
\hline 1.7 & 0.3 & 24 & 25.23 & $-1.349 \times 10^{-1}$ & $-7.800 \times 10^{-2}$ & $-3.547 \times 10^{-1}$ & $-2.153 \times 10^{-1}$ & $1.861 \times 10^{-1}$ & \\
\hline 1.8 & 0.3 & 0 & 0 & $-6.488 \times 10^{-2}$ & $-6.484 \times 10^{-2}$ & $-1.973 \times 10^{-1}$ & $-1.972 \times 10^{-1}$ & 0 & 0 \\
\hline 1.8 & 0.3 & 8 & & $-6.970 \times 10^{-2}$ & $-6.480 \times 10^{-2}$ & $-2.099 \times 10^{-1}$ & $-1.966 \times 10^{-1}$ & $5.206 \times 10^{-2}$ & $4.436 \times 10^{-2}$ \\
\hline 1.8 & 0.3 & 16 & 59628 & $-8.402 \times 10^{-2}$ & $-6.671 \times 10^{-2}$ & $-2.461 \times 10^{-1}$ & $-1.998 \times 10^{-1}$ & $9.857 \times 10^{-2}$ & $8.445 \times 10^{-2}$ \\
\hline 1.8 & 0.3 & 24 & 16 & $-1.075 \times 10^{-1}$ & $-7.030 \times 10^{-2}$ & $-3.026 \times 10^{-1}$ & $-2.056 \times 10^{-1}$ & $1.353 \times 10^{-1}$ & $1.169 \times 10^{-1}$ \\
\hline 1.8 & 0.3 & 32 & 33.1047 & $-1.404 \times 10^{-1}$ & $-8.153 \times 10^{-2}$ & $-3.762 \times 10^{-1}$ & $-2.255 \times 10^{-1}$ & $1.600 \times 10^{-1}$ & $1.394 \times 10^{-1}$ \\
\hline 1.9 & 0.3 & 0 & 0 & $-5.669 \times 10^{-2}$ & $-5.690 \times 10^{-2}$ & $-1.829 \times 10^{-1}$ & $-1.832 \times 10^{-1}$ & 0 & 0 \\
\hline 1.9 & 0.3 & 8 & 8. & $-6.010 \times 10^{-2}$ & $-5.683 \times 10^{-2}$ & $-1.922 \times 10^{-1}$ & $-1.824 \times 10^{-1}$ & $3.823 \times 10^{-2}$ & $3.229 \times 10^{-2}$ \\
\hline 1.9 & 0.3 & 16 & 6.8911 & $-7.025 \times 10^{-2}$ & $-5.818 \times 10^{-2}$ & $-2.189 \times 10^{-1}$ & $-1.844 \times 10^{-1}$ & $7.263 \times 10^{-2}$ & $6.165 \times 10^{-2}$ \\
\hline 1.9 & 0.3 & 24 & 25.0887 & $-8.701 \times 10^{-2}$ & $-6.054 \times 10^{-2}$ & $-2.609 \times 10^{-1}$ & $-1.874 \times 10^{-1}$ & $1.003 \times 10^{-1}$ & $8.579 \times 10^{-2}$ \\
\hline 1.9 & 0.3 & 32 & 33.0624 & $-1.106 \times 10^{-1}$ & $-6.912 \times 10^{-2}$ & $-3.157 \times 10^{-1}$ & $-2.034 \times 10^{-1}$ & $1.195 \times 10^{-1}$ & $1.032 \times 10^{-1}$ \\
\hline 2.0 & 0.3 & 0 & 0 & $-4.953 \times 10^{-2}$ & $-4.946 \times 10^{-2}$ & $-1.683 \times 10^{-1}$ & $-1.683 \times 10^{-1}$ & 0 & 0 \\
\hline 2.0 & 0.3 & 8 & 8.4616 & $-5.199 \times 10^{-2}$ & $-4.970 \times 10^{-2}$ & $-1.753 \times 10^{-1}$ & $-1.685 \times 10^{-1}$ & $2.862 \times 10^{-2}$ & $2.395 \times 10^{-2}$ \\
\hline 2.0 & 0.3 & 16 & 16.8262 & $-5.932 \times 10^{-2}$ & $-5.079 \times 10^{-2}$ & $-1.954 \times 10^{-1}$ & $-1.699 \times 10^{-1}$ & $5.452 \times 10^{-2}$ & $4.585 \times 10^{-2}$ \\
\hline 2.0 & 0.3 & 24 & 5.0215 & $-7.150 \times 10^{-2}$ & $-5.328 \times 10^{-2}$ & $-2.269 \times 10^{-1}$ & $-1.737 \times 10^{-1}$ & $7.564 \times 10^{-2}$ & $6.411 \times 10^{-2}$ \\
\hline 2.0 & 0.3 & 32 & 33.0172 & $-8.878 \times 10^{-2}$ & $-6.003 \times 10^{-2}$ & $-2.682 \times 10^{-1}$ & $-1.864 \times 10^{-1}$ & $9.077 \times 10^{-2}$ & $7.771 \times 10^{-2}$ \\
\hline
\end{tabular}


hole, and because the circular Teukolsky data used for the fits in Ref. [24] was computed for $3 M \leq p \leq 30 M$. As a first attempt to improve their behavior in the nearly horizon-skimming region, we have made fits using circular Teukolsky data for orbits with $M<p \leq 2 M$. In particular, for a black hole with $a=0.998 M$, we computed the circular Teukolsky-based fluxes $d L_{z} / d t$ and $d \iota / d t$ listed in Table I (columns 8 and 10). These results were fit (with error $\leqslant 0.2 \%$ ); see Eqs. (A4) and (A5) in the Appendix.
Despite using strong-field Teukolsky fluxes for our fit, we found fairly poor behavior of these rates of change, particularly as a function of eccentricity. To compensate for this, we introduced a kludge-type fit to correct the equatorial part of the flux, in addition to the circular part. We fit, as a function of $p$ and $e$, Teukolsky-based fluxes for $d E / d t$ and $d L_{z} / d t$ for orbits in the equatorial plane, and then introduce the following kludge fluxes for $E$ and $L_{z}$ :

TABLE IV. As in Tables II and III, but for different values of eccentricity $e$; the Teukolsky-based fluxes for $E$ and $L_{z}$ have an accuracy of $10^{-3}$.

\begin{tabular}{|c|c|c|c|c|c|c|c|c|c|}
\hline$\frac{p}{M}$ & $e$ & $\begin{array}{c}\theta_{\text {inc }} \\
\text { (deg.) }\end{array}$ & $\begin{array}{c}\iota \\
\text { (deg.) }\end{array}$ & $\begin{array}{l}\frac{d E}{d t} \times \frac{M^{2}}{\mu^{2}} \\
\text { (kludge) }\end{array}$ & $\begin{array}{c}\quad \frac{d E}{d t} \times \frac{M^{2}}{\mu^{2}} \\
\text { (Teukolsky) }\end{array}$ & $\begin{array}{l}\frac{d L_{z}}{d t} \times \frac{M}{\mu^{2}} \\
\text { (kludge) }\end{array}$ & $\begin{array}{c}\frac{d L_{z}}{d t} \times \frac{M}{\mu^{2}} \\
\text { (Teukolsky) }\end{array}$ & $\begin{array}{l}\frac{d \iota}{d t} \times \frac{M}{\mu^{2}} \\
\text { (kludge) }\end{array}$ & $\begin{array}{l}\frac{d \theta_{\text {inc }}}{d t} \times \frac{M}{\mu^{2}} \\
\text { (kludge) }\end{array}$ \\
\hline 1.6 & 0.4 & 0 & 0 & $66 \times$ & $-7.772 \times 10$ & $-1.918 \times 10$ & $-1.919 \times 10$ & 0 & 0 \\
\hline 1.6 & .4 & 8 & 5863 & $9.433 \times 10^{-2}$ & $-7.645 \times 10^{-2}$ & $-2.297 \times 10^{-1}$ & $-1.881 \times 10^{-1}$ & $1.528 \times 10^{-1}$ & $1.370 \times 10^{-1}$ \\
\hline 1.6 & 0.4 & 16 & 17.0151 & $1.432 \times 10^{-1}$ & $-7.651 \times 10^{-2}$ & $-3.382 \times 10^{-1}$ & $-1.837 \times 10^{-1}$ & $2.873 \times 10$ & $2.584 \times 10^{-1}$ \\
\hline 1.7 & 0.4 & 0 & 0 & $-7.882 \times 10^{-2}$ & $-7.953 \times 10^{-2}$ & $-2.097 \times 10^{-1}$ & $-2.115 \times 10^{-1}$ & 0 & 0 \\
\hline 1.7 & 0.4 & 8 & 426 & $9.002 \times 10^{-2}$ & $-7.408 \times 10^{-2}$ & $-2.367 \times 10^{-1}$ & $-1.978 \times 10^{-1}$ & $1.087 \times 1$ & $9.656 \times 10^{-2}$ \\
\hline 1.7 & 0.4 & 16 & 16.9502 & $-1.229 \times 10^{-1}$ & $-7.682 \times 10^{-2}$ & $-3.143 \times 10^{-1}$ & $-2.025 \times 10^{-1}$ & $2.054 \times 10^{-1}$ & $1.830 \times 10^{-1}$ \\
\hline 1.7 & 0.4 & 24 & 1282 & $-1.760 \times 10^{-1}$ & $-8.090 \times 10^{-2}$ & $-4.336 \times 10^{-1}$ & $-2.075 \times 10^{-1}$ & $2.809 \times 10^{-1}$ & $2.514 \times 10^{-1}$ \\
\hline 1.8 & 0.4 & 0 & 0 & $-7.107 \times 10^{-2}$ & $-7.007 \times 10^{-2}$ & $-2.013 \times 10^{-1}$ & $-1.988 \times 10^{-1}$ & 0 & 0 \\
\hline 1.8 & 0.4 & 8 & & $-7.877 \times 10^{-2}$ & $-7.001 \times 10^{-2}$ & $-2.209 \times 10^{-1}$ & $-1.981 \times 10^{-1}$ & $7.788 \times 10^{-2}$ & $6.879 \times 10^{-2}$ \\
\hline 1.8 & 0.4 & 16 & 17 & $-1.015 \times 10^{-1}$ & $-7.009 \times 10^{-2}$ & $-2.774 \times 10^{-1}$ & $-1.965 \times 10^{-1}$ & $1.478 \times 1$ & $1.309 \times 10^{-1}$ \\
\hline 1.8 & 0.4 & 24 & 25.0646 & $-1.383 \times 10^{-1}$ & $-7.314 \times 10^{-2}$ & $-3.646 \times 10^{-1}$ & $-2.003 \times 10^{-1}$ & $2.036 \times 1$ & $1.810 \times 10^{-1}$ \\
\hline 1.8 & 0.4 & 32 & 0184 & $-1.887 \times 1$ & $-9.193 \times 10^{-2}$ & $-4.755 \times 1$ & $-2.319 \times 1$ & $2.414 \times 1$ & $2.156 \times 10^{-1}$ \\
\hline 1.9 & 0.4 & 0 & 0 & $-6.187 \times 10^{-2}$ & $-6.267 \times 10^{-2}$ & $-1.861 \times 10^{-1}$ & $-1.881 \times 10^{-1}$ & 0 & 0 \\
\hline 1.9 & 0.4 & 8 & & $-6.728 \times 1$ & $-6.216 \times 10^{-2}$ & $-2.006 \times 1$ & -1.8 & $5.666 \times$ & $4.980 \times 10^{-2}$ \\
\hline 1.9 & 0.4 & 16 & 8173 & $-8.328 \times 10^{-2}$ & $-6.222 \times 10^{-2}$ & $-2.424 \times 10^{-1}$ & $-1.844 \times 10^{-1}$ & $1.079 \times 1$ & $9.506 \times 10^{-2}$ \\
\hline 1.9 & 0.4 & 24 & 0006 & $-1.094 \times 10^{-1}$ & $-6.486 \times 10^{-2}$ & $-3.071 \times 10^{-1}$ & $-1.878 \times 1$ & $1.495 \times 1$ & $1.322 \times 10^{-1}$ \\
\hline 1.9 & 0.4 & 32 & 9804 & $-1.452 \times 10^{-1}$ & $-7.884 \times 10^{-2}$ & $-3.896 \times 10^{-1}$ & $-2.158 \times 10^{-1}$ & $1.787 \times 10^{-1}$ & $1.588 \times 10^{-1}$ \\
\hline 2.0 & 0.4 & 0 & 0 & $-5.483 \times 10^{-2}$ & $-5.457 \times 10^{-2}$ & $-1.735 \times 10^{-1}$ & $-1.729 \times 10^{-1}$ & 0 & 0 \\
\hline 2.0 & 0.4 & 8 & & & $-5.445 \times 10^{-2}$ & $-1.844 \times 10^{-1}$ & $-1.720 \times 10^{-1}$ & $4.222 \times$ & $3.686 \times 10^{-2}$ \\
\hline 2.0 & 0.4 & 16 & 36 & $-7.020 \times 10^{-2}$ & $-5.555 \times 10^{-2}$ & $-2.158 \times 10^{-1}$ & $-1.733 \times 10^{-1}$ & $8.064 \times 1$ & $7.057 \times 10^{-2}$ \\
\hline 2.0 & 0.4 & 24 & & $-8.902 \times 10^{-2}$ & $-5.844 \times 10^{-2}$ & $-2.645 \times 11$ & $-1.778 \times 1$ & $1.122 \times 1$ & $9.860 \times 10^{-2}$ \\
\hline 2.0 & 0.4 & 2 & & $-1.150 \times 10^{-1}$ & $-6.536 \times 10^{-2}$ & $-3.267 \times 10^{-1}$ & $-1.896 \times 10^{-1}$ & $1.351 \times 10$ & $1.193 \times 10^{-1}$ \\
\hline .7 & & 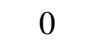 & 0 & $-7.421 \times 10^{-2}$ & $-7.401 \times 10^{-2}$ & $-1.815 \times 10^{-1}$ & $-1.810 \times 10^{-1}$ & 0 & 0 \\
\hline 1.7 & 0.5 & 8 & 736 & $-8.957 \times 10^{-2}$ & $-7.168 \times 10^{-2}$ & $-2.173 \times 10^{-1}$ & $-1.750 \times 10^{-1}$ & $1.379 \times 1$ & $1.256 \times 10^{-1}$ \\
\hline 1.7 & 0.5 & 16 & 16.8300 & $-1.347 \times 10^{-1}$ & $-6.999 \times 10^{-2}$ & $-3.201 \times 10^{-1}$ & $-1.676 \times 10^{-1}$ & $2.611 \times 10^{-1}$ & $2.378 \times 10^{-1}$ \\
\hline 1.8 & 0.5 & 0 & 0 & $-7.589 \times 10^{-2}$ & $-7.620 \times 10^{-2}$ & $-1.993 \times 10^{-1}$ & $-2.000 \times 10^{-1}$ & 0 & 0 \\
\hline 1.8 & 0.5 & 8 & & $-8.644 \times 10^{-2}$ & $-6.929 \times 10^{-2}$ & $-2.254 \times 10^{-1}$ & $-1.829 \times 10^{-1}$ & $05 \times$ & $9.076 \times$ \\
\hline 1.8 & 0.5 & 16 & 7776 & $-1.175 \times 10^{-1}$ & $-7.210 \times 10^{-2}$ & $-3.004 \times 10^{-1}$ & $-1.880 \times 10^{-1}$ & $1.911 \times 10^{-1}$ & $1.726 \times 10^{-1}$ \\
\hline 1.8 & 0.5 & 24 & 24.9413 & $-1.678 \times 10^{-1}$ & $-7.395 \times 10^{-2}$ & $-4.158 \times 10^{-1}$ & $-1.881 \times 10^{-1}$ & $2.638 \times 10^{-1}$ & $2.385 \times 10^{-1}$ \\
\hline 1.9 & 0.5 & 0 & 0 & $-6.646 \times 10^{-2}$ & $-6.620 \times 10^{-2}$ & $-1.855 \times 10^{-1}$ & $-1.849 \times 10^{-1}$ & 0 & 0 \\
\hline 1.9 & 0.5 & 8 & 059 & $-7.386 \times 10^{-2}$ & $-6.320 \times 10^{-2}$ & $-2.048 \times 10^{-1}$ & $-1.768 \times 10^{-1}$ & $7.312 \times 1$ & $6.579 \times 10^{-2}$ \\
\hline 1.9 & 0.5 & 16 & .7233 & $-9.572 \times 10^{-2}$ & $-6.551 \times 10^{-2}$ & $-2.603 \times 10^{-1}$ & $-1.809 \times 10^{-1}$ & $1.395 \times 10^{-1}$ & $1.255 \times 10^{-1}$ \\
\hline 1.9 & 0.5 & 24 & 8877 & $-1.312 \times 10^{-1}$ & $-7.087 \times 10^{-2}$ & $-3.461 \times 10^{-1}$ & $-1.909 \times 10^{-1}$ & $1.937 \times 10^{-1}$ & $1.744 \times 10^{-1}$ \\
\hline 1.9 & 0.5 & 32 & 32.8741 & $-1.795 \times 10^{-1}$ & $-8.247 \times 10^{-2}$ & $-4.544 \times 10^{-1}$ & $-2.091 \times 10^{-1}$ & $2.320 \times 10^{-1}$ & $2.092 \times 10^{-1}$ \\
\hline 2.0 & 0.5 & 0 & 0 & $-5.987 \times 10^{-2}$ & $-5.995 \times 10^{-2}$ & $-1.761 \times 10^{-1}$ & $-1.763 \times 10^{-1}$ & 0 & 0 \\
\hline 2.0 & 0.5 & 8 & & $-6.516 \times 10^{-2}$ & $-5.918 \times 10^{-2}$ & $-1.906 \times 10^{-1}$ & $-1.738 \times 10^{-1}$ & $5.456 \times 10^{-2}$ & $4.882 \times 10^{-2}$ \\
\hline 2.0 & 0.5 & 16 & 6.6725 & $-8.081 \times 10^{-2}$ & $-5.817 \times 10^{-2}$ & $-2.324 \times 10^{-1}$ & $-1.694 \times 10^{-1}$ & $1.044 \times 10^{-1}$ & $9.343 \times 10^{-2}$ \\
\hline 2.0 & 0.5 & 24 & 24.8347 & $-1.063 \times 10^{-1}$ & $-6.254 \times 10^{-2}$ & $-2.970 \times 10^{-1}$ & $-1.776 \times 10^{-1}$ & $1.456 \times 10^{-1}$ & $1.304 \times 10^{-1}$ \\
\hline 2.0 & 0.5 & 32 & 32.8378 & $-1.412 \times 10^{-1}$ & $-6.993 \times 10^{-2}$ & $-3.787 \times 10^{-1}$ & $-1.893 \times 10^{-1}$ & $1.756 \times 10^{-1}$ & $1.576 \times 10^{-1}$ \\
\hline
\end{tabular}




$$
\begin{aligned}
\frac{d E}{d t}(p, e, \iota)= & \left(\frac{d E}{d t}\right)_{\mathrm{GG}}(p, e, \iota)-\left(\frac{d E}{d t}\right)_{\mathrm{GG}}(p, e, 0) \\
& +\left(\frac{d E}{d t}\right)_{\mathrm{fit} \mathrm{eq}}(p, e),
\end{aligned}
$$

$$
\begin{aligned}
\frac{d L_{z}}{d t}(p, e, \iota)= & \left(\frac{d L_{z}}{d t}\right)_{\mathrm{GG}}(p, e, \iota)-\left(\frac{d L_{z}}{d t}\right)_{\mathrm{GG}}(p, e, 0) \\
& +\left(\frac{d L_{z}}{d t}\right)_{\mathrm{fit} \mathrm{eq}}(p, e) .
\end{aligned}
$$

[Note that Eq. (40) for $d Q / d t$ is not modified by this procedure since $d Q / d t=0$ for equatorial orbits.] Using equatorial noncircular Teukolsky data provided by Drasco [21,33] for $a=0.998$ and $M<p \leq 2 M$ (the $\iota=0$ Teukolsky data in Tables II, III, and IV), we found fits (with error $\lesssim 1.5 \%$ ); see Eqs. (A8) and (A9). Note that the fits for equatorial fluxes are significantly less accurate than the fits for circular fluxes. This appears to be due to the fact that, close to the black hole, many harmonics are needed in order for the Teukolsky-based fluxes to converge, especially for eccentric orbits (cf. Figs. 2 and 3 of Ref. [21], noting the number of radial harmonics that have significant contribution to the flux). Truncation of these sums is likely a source of some error in the fluxes themselves, making it difficult to make a fit of as high quality as we could in the circular case.

TABLE V. Variation in the inclination angles $\iota$ and $\theta_{\text {inc }}$ as well as time needed to reach the separatrix for several inspirals through the nearly horizon-skimming regime. In all of these cases, the binary's mass ratio was fixed to $\mu / M=10^{-6}$, the large black hole's spin was fixed to $a=0.998 M$, and the orbits were begun at $p=1.9 M$. The time interval $\Delta t$ is the total accumulated time it takes for the inspiralling body to reach the separatrix (at which time it rapidly plunges into the black hole). The angles $\Delta \theta_{\text {inc }}$ and $\Delta \iota$ are the total integrated change in these inclination angles that we compute. For the $e=0$ cases, inspirals are computed using fits to the circular

\begin{tabular}{|c|c|c|c|c|c|}
\hline$e$ & $\theta_{\text {inc }}($ deg. $)$ & $\iota$ (deg.) & $\Delta t / M$ & $\Delta \theta_{\text {inc }}$ (deg.) & $\Delta \iota$ (deg.) \\
\hline 0 & 0 & 0 & $1.250 \times 10^{6}$ & 0 & 0 \\
\hline 0 & 5 & 5.355510 & $1.217 \times 10^{6}$ & $1.949 \times 10^{-1}$ & $4.954 \times 10^{-1}$ \\
\hline 0 & 10 & 10.679331 & $1.118 \times 10^{6}$ & $3.468 \times 10^{-1}$ & $8.631 \times 10^{-1}$ \\
\hline 0 & 15 & 15.943192 & $9.574 \times 10^{5}$ & $4.236 \times 10^{-1}$ & 1.019 \\
\hline 0 & 20 & 21.125167 & $7.446 \times 10^{5}$ & $4.109 \times 10^{-1}$ & $9.440 \times 10^{-1}$ \\
\hline 0 & 25 & 26.211779 & $4.981 \times 10^{5}$ & $3.158 \times 10^{-1}$ & $6.860 \times 10^{-1}$ \\
\hline 0 & 30 & 31.199048 & $2.528 \times 10^{5}$ & $1.732 \times 10^{-1}$ & $3.527 \times 10^{-1}$ \\
\hline 0 & 35 & 36.092514 & $6.584 \times 10^{4}$ & $4.636 \times 10^{-2}$ & $8.806 \times 10^{-2}$ \\
\hline 0.1 & 0 & 0 & $1.228 \times 10^{6}$ & 0 & 0 \\
\hline 0.1 & 5 & 5.351602 & $1.198 \times 10^{6}$ & $4.517 \times 10^{-1}$ & $7.766 \times 10^{-1}$ \\
\hline 0.1 & 10 & 10.671900 & $1.103 \times 10^{6}$ & $6.900 \times 10^{-1}$ & 1.236 \\
\hline 0.1 & 15 & 15.932962 & $9.426 \times 10^{5}$ & $7.283 \times 10^{-1}$ & 1.344 \\
\hline 0.1 & 20 & 21.113129 & $7.315 \times 10^{5}$ & $6.433 \times 10^{-1}$ & 1.187 \\
\hline 0.1 & 25 & 26.199088 & $4.900 \times 10^{5}$ & $4.780 \times 10^{-1}$ & $8.547 \times 10^{-1}$ \\
\hline 0.1 & 30 & 31.186915 & $2.513 \times 10^{5}$ & $2.730 \times 10^{-1}$ & $4.585 \times 10^{-1}$ \\
\hline 0.1 & 35 & 36.082095 & $6.589 \times 10^{4}$ & $8.385 \times 10^{-2}$ & $1.279 \times 10^{-1}$ \\
\hline 0.2 & 0 & 0 & $1.173 \times 10^{6}$ & 0 & 0 \\
\hline 0.2 & 5 & 5.339916 & $1.150 \times 10^{6}$ & 1.204 & 1.598 \\
\hline 0.2 & 10 & 10.649670 & $1.064 \times 10^{6}$ & 1.698 & 2.331 \\
\hline 0.2 & 15 & 15.902348 & $9.043 \times 10^{5}$ & 1.618 & 2.293 \\
\hline 0.2 & 20 & 21.077081 & $6.980 \times 10^{5}$ & 1.324 & 1.900 \\
\hline 0.2 & 25 & 26.161046 & $4.693 \times 10^{5}$ & $9.545 \times 10^{-1}$ & 1.351 \\
\hline 0.2 & 30 & 31.150481 & $2.486 \times 10^{5}$ & $5.674 \times 10^{-1}$ & $7.711 \times 10^{-1}$ \\
\hline 0.2 & 35 & 36.050712 & $7.562 \times 10^{4}$ & $2.070 \times 10^{-1}$ & $2.648 \times 10^{-1}$ \\
\hline 0.3 & 0 & 0 & $1.087 \times 10^{6}$ & 0 & 0 \\
\hline 0.3 & 5 & 5.320559 & $1.069 \times 10^{6}$ & 2.307 & 2.788 \\
\hline 0.3 & 10 & 10.612831 & $1.001 \times 10^{6}$ & 3.256 & 4.007 \\
\hline 0.3 & 15 & 15.851572 & $8.454 \times 10^{5}$ & 2.984 & 3.741 \\
\hline 0.3 & 20 & 21.017212 & $6.483 \times 10^{5}$ & 2.375 & 2.998 \\
\hline 0.3 & 25 & 26.097732 & $4.408 \times 10^{5}$ & 1.700 & 2.129 \\
\hline 0.3 & 30 & 31.089639 & $2.493 \times 10^{5}$ & 1.040 & 1.276 \\
\hline 0.3 & 35 & 35.997987 & $1.108 \times 10^{5}$ & $4.626 \times 10^{-1}$ & $5.569 \times 10^{-1}$ \\
\hline
\end{tabular}
Teukolsky fluxes of $E$ and $L_{z}$; for eccentric orbits we use the kludge fluxes (40), (43), and (44). Notice that $\Delta \theta_{\text {inc }}$ and $\Delta \iota$ are always positive - the inclination angle always increases during the inspiral through the nearly horizon-skimming region. The magnitude of this increase never exceeds a few degrees. 
These fits were then finally used in Eqs. (43) and (44) to calculate the kludge fluxes $d E / d t$ and $d L_{z} / d t$ for generic orbits. This kludge reproduces to high accuracy our fits to the Teukolsky-based fluxes for circular orbits $(e=0)$ or equatorial orbits $(\iota=0)$. Some residual error remains because the $\iota=0$ limit of the circular fits do not precisely equal the $e=0$ limit of the equatorial fits.

Table I compares our kludge to Teukolsky-based fluxes for circular orbits; the two methods agree to several digits. Tables II, III, and IV compare our kludge to the generic Teukolsky-based fluxes for $d E / d t$ and $d L_{z} / d t$ provided by Drasco [21,33]. In all cases, the kludge fluxes $d E / d t$ and $d L_{z} / d t$ have the correct qualitative behavior, being negative for all the orbital parameters under consideration $(a=$ $0.998 M, 1<p / M \leq 2,0 \leq e \leq 0.5$ and $0^{\circ} \leq \iota \leq 41^{\circ}$ ). The relative difference between the kludge and Teukolsky fluxes is always less than $25 \%$ for $e=0$ and $e=0.1$ (even for orbits very close to separatrix). The accuracy remains good at larger eccentricity, though it degrades somewhat as orbits come close to the separatrix.

Tables I, II, III, and IV also present the kludge values of the fluxes $d \iota / d t$ and $d \theta_{\text {inc }} / d t$ as computed using Eqs. (43) and (44) for $d E / d t$ and $d L_{z} / d t$, plus Eq. (40) for $d Q / d t$. Though certainly not the last word on inclination evolution (pending rigorous computation of $d Q / d t$ ), these rates of change probably represent a better approximation than results published to date in the literature. (Indeed, prior work has often used the crude approximation $d \iota / d t=0$ [21] to estimate $d Q / d t$ given $d E / d t$ and $d L_{z} / d t$.)

Most significantly, we find that $(d \iota / d t)_{\text {kludge }}>0$ and $\left(d \theta_{\text {inc }} / d t\right)_{\text {kludge }}>0$ for all of the orbital parameters we consider. In other words, we find that $d \iota / d t$ and $d \theta_{\text {inc }} / d t$ do not ever change sign.

Finally, in Table V we compute the changes in $\theta_{\text {inc }}$ and $\iota$ for the inspiral with mass ratio $\mu / M=10^{-6}$. In all cases, we start at $p / M=1.9$. The small body then inspirals through the nearly horizon-skimming region until it reaches the separatrix; at this point, the small body will fall into the large black hole on a dynamical timescale $\sim M$, so we terminate the calculation. The evolution of circular orbits is computed using our fits to the circular Teukolsky fluxes of $E$ and $L_{z}$; for eccentric orbits we use the kludge fluxes (40), (43), and (44). As this exercise demonstrates, the change in inclination during inspiral is never larger than a few degrees. Not only is there no unique sign change in the nearly horizon-skimming region, but the magnitude of the inclination change remains puny. This leaves little room for the possibility that this class of orbits may have a clear observational imprint on the EMRI waveforms to be detected by LISA.

\section{CONCLUSIONS}

We have performed a detailed analysis of the orbital motion near the horizon of near-extremal Kerr black holes. We have demonstrated the existence of a class of orbits, which we have named "noncircular nearly horizonskimming orbits," for which the angular momentum $L_{z}$ increases with the orbit's inclination, while keeping latus rectum and eccentricity fixed. This behavior, in stark contrast to that of Newtonian orbits, generalizes earlier results for circular orbits [14].

Furthermore, to assess whether this class of orbits can produce a unique imprint on EMRI waveforms (an important source for future LISA observations), we have studied, in the adiabatic approximation, the radiative evolution of inclination angle for a small body orbiting in the nearly horizon-skimming region. For circular orbits, we have reexamined the analysis of Ref. [14] using an improved code for computing Teukolsky-based fluxes of the energy and angular momentum. Significantly correcting Ref. [14]'s results, we found no decrease in the orbit's inclination angle. Inclination always increases during inspiral.

We next carried out such an analysis for eccentric nearly horizon-skimming orbits. In this case, we used kludge fluxes to evolve the constants of motion $E, L_{z}$ and $Q$ [24]. We find that these fluxes are fairly accurate when compared with the available Teukolsky-based fluxes, indicating that they should provide at least qualitatively correct information regarding inclination evolution. As for circular orbits, we find that the orbit's inclination never decreases. For both circular and noncircular configurations, we find that the magnitude of the inclination change is quite paltry - only a few degrees at most.

Quite generically, therefore, we found that the inclination angle of both circular and eccentric nearly horizonskimming orbits never decreases during the inspiral. Revising the results obtained in Ref. [14], we thus conclude that such orbits are not likely to yield a peculiar, unique imprint on the EMRI waveforms detectable by LISA.

\section{ACKNOWLEDGMENTS}

It is a pleasure to thank Kostas Glampedakis for enlightening comments and advice, and Steve Drasco for useful discussions and for also providing the noncircular Teukolsky data that we used in this paper. The supercomputers used in this investigation were provided by funding from the JPL Office of the Chief Information Officer. This work was supported in part by the DFG Grant No. SFB TR/ 7, by NASA Grant No. NNG05G105G, and by NSF Grant No. PHY-0449884. S. A. H. gratefully acknowledges support from the MIT Class of 1956 Career Development Fund.

\section{APPENDIX}

In this Appendix we report the expressions for the postNewtonian fluxes and the fits to the Teukolsky data necessary to compute the kludge fluxes introduced in Sec. V. In 
particular the $2 \mathrm{PN}$ fluxes are given by [24]

$$
\begin{aligned}
\left(\frac{d E}{d t}\right)_{2 \mathrm{PN}}= & -\frac{32}{5} \frac{\mu^{2}}{M^{2}}\left(\frac{M}{p}\right)^{5}\left(1-e^{2}\right)^{3 / 2}\left[g_{1}(e)-\tilde{a}\left(\frac{M}{p}\right)^{3 / 2} g_{2}(e) \cos \iota-\left(\frac{M}{p}\right) g_{3}(e)+\pi\left(\frac{M}{p}\right)^{3 / 2} g_{4}(e)\right. \\
& \left.-\left(\frac{M}{p}\right)^{2} g_{5}(e)+\tilde{a}^{2}\left(\frac{M}{p}\right)^{2} g_{6}(e)-\frac{527}{96} \tilde{a}^{2}\left(\frac{M}{p}\right)^{2} \sin ^{2} \iota\right] \\
\left(\frac{d L_{z}}{d t}\right)_{2 \mathrm{PN}}= & -\frac{32}{5} \frac{\mu^{2}}{M}\left(\frac{M}{p}\right)^{7 / 2}\left(1-e^{2}\right)^{3 / 2}\left[g_{9}(e) \cos \iota+\tilde{a}\left(\frac{M}{p}\right)^{3 / 2}\left(g_{10}^{a}(e)-\cos ^{2} \iota g_{10}^{b}(e)\right)-\left(\frac{M}{p}\right) g_{11}(e) \cos \iota\right. \\
& \left.+\pi\left(\frac{M}{p}\right)^{3 / 2} g_{12}(e) \cos \iota-\left(\frac{M}{p}\right)^{2} g_{13}(e) \cos \iota+\tilde{a}^{2}\left(\frac{M}{p}\right)^{2} \cos \iota\left(g_{14}(e)-\frac{45}{8} \sin ^{2} \iota\right)\right], \\
\left(\frac{d Q}{d t}\right)_{2 \mathrm{PN}}= & -\frac{64}{5} \frac{\mu^{2}}{M}\left(\frac{M}{p}\right)^{7 / 2} \sqrt{Q} \sin \iota\left(1-e^{2}\right)^{3 / 2}\left[g_{9}(e)-\tilde{a}\left(\frac{M}{p}\right)^{3 / 2} \cos \iota g_{10}^{b}(e)-\left(\frac{M}{p}\right) g_{11}(e)+\pi\left(\frac{M}{p}\right)^{3 / 2} g_{12}(e)\right. \\
& \left.-\left(\frac{M}{p}\right)^{2} g_{13}(e)+\tilde{a}^{2}\left(\frac{M}{p}\right)^{2} \times\left(g_{14}(e)-\frac{45}{8} \sin ^{2} \iota\right)\right]
\end{aligned}
$$

where $\mu$ is the mass of the infalling body and where the various $e$-dependent coefficients are

$$
\begin{gathered}
g_{1}(e) \equiv 1+\frac{73}{24} e^{2}+\frac{37}{96} e^{4}, \quad g_{2}(e) \equiv \frac{73}{12}+\frac{823}{24} e^{2}+\frac{949}{32} e^{4}+\frac{491}{192} e^{6}, \quad g_{3}(e) \equiv \frac{1247}{336}+\frac{9181}{672} e^{2}, \\
g_{4}(e) \equiv 4+\frac{1375}{48} e^{2}, \quad g_{5}(e) \equiv \frac{44711}{9072}+\frac{172157}{2592} e^{2}, \quad g_{6}(e) \equiv \frac{33}{16}+\frac{359}{32} e^{2}, \quad g_{9}(e) \equiv 1+\frac{7}{8} e^{2}, \\
g_{10}^{a}(e) \equiv \frac{61}{24}+\frac{63}{8} e^{2}+\frac{95}{64} e^{4}, \quad g_{10}^{b}(e) \equiv \frac{61}{8}+\frac{91}{4} e^{2}+\frac{461}{64} e^{4}, \quad g_{11}(e) \equiv \frac{1247}{336}+\frac{425}{336} e^{2}, \\
g_{12}(e) \equiv 4+\frac{97}{8} e^{2}, \quad g_{13}(e) \equiv \frac{44711}{9072}+\frac{302893}{6048} e^{2}, \quad g_{14}(e) \equiv \frac{33}{16}+\frac{95}{16} e^{2} .
\end{gathered}
$$

The fits to the circular Teukolsky data of Table I are instead given by

$$
\begin{aligned}
\left(\frac{d L_{z}}{d t}\right)_{\text {fit circ }}(p, \iota)= & -\frac{32}{5} \frac{\mu^{2}}{M}\left(\frac{M}{p}\right)^{7 / 2}\left\{\cos \iota+\left(\frac{M}{p}\right)^{3 / 2}\left(\frac{61}{24}-\frac{61}{8} \cos ^{2} \iota+4 \pi \cos \iota\right)-\frac{1247}{336}\left(\frac{M}{p}\right) \cos \iota\right. \\
+ & \left(\frac{M}{p}\right)^{2} \cos \iota\left(-\frac{1625}{567}-\frac{45}{8} \sin ^{2} \iota\right)+\left(\frac{M}{p}\right)^{5 / 2}\left[\tilde{d}_{1}(p / M)+\tilde{d}_{2}(p / M) \cos \iota+\tilde{d}_{3}(p / M) \cos ^{2} \iota\right. \\
+ & \left.\left.\tilde{d}_{4}(p / M) \cos ^{3} \iota+\tilde{d}_{5}(p / M) \cos ^{4} \iota+\tilde{d}_{6}(p / M) \cos ^{5} \iota+\cos \iota\left(\frac{M}{p}\right)^{3 / 2}\left(A+B \cos ^{2} \iota\right)\right]\right\}, \\
\left(\frac{d \iota}{d t}\right)_{\text {fit circ }}(p, \iota)= & \frac{32}{5} \frac{\mu^{2}}{M} \frac{\sin ^{2} \iota}{\sqrt{Q(p, 0, \iota)}}\left(\frac{M}{p}\right)^{5}\left\{\frac{61}{24}+\left(\frac{M}{p}\right) \tilde{d}_{1}(p / M)+\cos \iota\left(\frac{M}{p}\right)^{1 / 2}\left[a_{d}^{7}+b_{d}^{7}\left(\frac{M}{p}\right)+c_{d}^{7}\left(\frac{M}{p}\right)^{3 / 2}\right]\right. \\
& \left.+\cos ^{2} \iota\left(\frac{M}{p}\right) \tilde{d}_{8}(p / M)+\cos \iota\left(\frac{M}{p}\right)^{5 / 2}\left[\tilde{h}_{1}(p / M)+\cos ^{2} \iota \tilde{h}_{2}(p / M)\right]\right\},
\end{aligned}
$$

where

$$
\tilde{d}_{i}(x) \equiv a_{d}^{i}+b_{d}^{i} x^{-1 / 2}+c_{d}^{i} x^{-1}, \quad i=1, \ldots, 8, \quad \tilde{h}_{i}(x) \equiv a_{h}^{i}+b_{h}^{i} x^{-1 / 2}, \quad i=1,2
$$

and the numerical coefficients are given by 


$$
a_{h}^{1}=-278.9387, \quad b_{h}^{1}=84.1414, \quad a_{h}^{2}=8.6679, \quad b_{h}^{2}=-9.2401, \quad A=-18.3362, \quad B=24.9034,
$$

and by the following table.

\begin{tabular}{|c|c|c|c|c|c|c|c|c|}
\hline$i$ & 1 & 2 & 3 & 4 & 5 & 6 & 7 & 8 \\
\hline$a_{d}^{i}$ & 15.8363 & 445.4418 & -2027.7797 & 3089.1709 & -2045.2248 & 498.6411 & -8.7220 & 50.8345 \\
\hline$b_{d}^{i}$ & -55.6777 & -1333.2461 & 5940.4831 & -9103.4472 & 6113.1165 & -1515.8506 & -50.8950 & -131.6422 \\
\hline$c_{d}^{i}$ & 38.6405 & 1049.5637 & -4513.0879 & 6926.3191 & -4714.9633 & 1183.5875 & 251.4025 & 83.0834 \\
\hline
\end{tabular}

Note that the functional form of these fits was obtained from Eqs. (57) and (58) of Ref. [24] by setting $\tilde{a}$ (i.e., $q$ in their notation) to 1. Finally, we give expressions for the fits to the equatorial Teukolsky data of Tables II, III, and IV (data with $\iota=0$, columns with header Teukolsky):

$$
\begin{aligned}
\left(\frac{d E}{d t}\right)_{\mathrm{fit} \mathrm{eq}}(p, e)= & \left(\frac{d E}{d t}\right)_{2 \mathrm{PN}}(p, e, 0)-\frac{32}{5}\left(\frac{\mu}{M}\right)^{2}\left(\frac{M}{p}\right)^{5}\left(1-e^{2}\right)^{3 / 2}\left[\tilde{g}_{1}(e)+\tilde{g}_{2}(e)\left(\frac{M}{p}\right)^{1 / 2}+\tilde{g}_{3}(e)\left(\frac{M}{p}\right)\right. \\
& \left.+\tilde{g}_{4}(e)\left(\frac{M}{p}\right)^{3 / 2}+\tilde{g}_{5}(e)\left(\frac{M}{p}\right)^{2}\right] \\
\left(\frac{L_{z}}{d t}\right)_{\mathrm{fit} \mathrm{eq}}(p, e)= & \left(\frac{L_{z}}{d t}\right)_{2 \mathrm{PN}}(p, e, 0)-\frac{32}{5} \frac{\mu^{2}}{M}\left(\frac{M}{p}\right)^{7 / 2}\left(1-e^{2}\right)^{3 / 2}\left[\tilde{f}_{1}(e)+\tilde{f}_{2}(e)\left(\frac{M}{p}\right)^{1 / 2}\right. \\
& \left.+\tilde{f}_{3}(e)\left(\frac{M}{p}\right)+\tilde{f}_{4}(e)\left(\frac{M}{p}\right)^{3 / 2}+\tilde{f}_{5}(e)\left(\frac{M}{p}\right)^{2}\right]
\end{aligned}
$$

\begin{tabular}{|c|c|c|c|c|c|c|c|c|}
\hline & $a_{g}^{i}$ & $b_{g}^{i}$ & $c_{g}^{i}$ & $d_{g}^{i}$ & $a_{f}^{i}$ & $b_{f}^{i}$ & $c_{f}^{i}$ & $d_{f}^{i}$ \\
\hline 1 & 6.4590 & -2038.7301 & 6639.9843 & 227709.2187 & 5.4577 & -3116.4034 & 4711.7065 & 214332.2907 \\
\hline 2 & -31.2215 & 10390.6778 & -27505.7295 & -1224376.5294 & -26.6519 & 15958.6191 & -16390.4868 & -1147201.4687 \\
\hline 3 & 57.1208 & -19800.4891 & 39527.8397 & 2463977.3622 & 50.4374 & -30579.3129 & 15749.9411 & 2296989.5466 \\
\hline . & -49.7051 & 16684.4629 & -21714.7941 & -2199231.9494 & -46.7816 & 25968.8743 & 656.3460 & -2038650.9838 \\
\hline 5 & 16.4697 & -5234.2077 & 2936.2391 & 734454.5696 & 15.6660 & -8226.3892 & -4903.9260 & 676553.2755 \\
\hline
\end{tabular}

where the numerical coefficients are given by the following table.

[1] http://lisa.nasa.gov/; http://sci.esa.int/home/lisa/.

[2] J. Kormendy and D. Richstone, Annu. Rev. Astron. Astrophys. 33, 581 (1995).

[3] J. R. Gair, L. Barack, T. Creighton, C. Cutler, S. L. Larson, E. S. Phinney, and M. Vallisneri, Classical Quantum Gravity 21, S1595 (2004).

[4] S. L. Shapiro, Astrophys. J. 620, 59 (2005).

[5] L. Rezzolla, T. W. Maccarone, S. Yoshida, and O. Zanotti, Mon. Not. R. Astron. Soc. 344, L37 (2003).

[6] R. Shafee, J. E. McClintock, R. Narayan, S. W. Davis, L.X. Li, and R.A. Remilland, Astrophys. J. 636, L113 (2006).
[7] J.E. McClintock, R. Shafee, R. Narayan, R. A. Remilland, S. W. Davis, and L.-X. Li, Astrophys. J. 652, 518 (2006).

[8] A. C. Fabian and G. Miniutti, in Kerr Spacetime: Rotating Black Holes in General Relativity, 2005, edited by D.L. Wiltshire, M. Visser, and S.M. Scott (to be published); arXiv:astro-ph/0507409.

[9] L. W. Brenneman and C. S. Reynolds, Astrophys. J. 652, 1028 (2006).

[10] L. Barack and C. Cutler, Phys. Rev. D 69, 082005 (2004).

[11] M. Volonteri, P. Madau, E. Quataert, and M. J. Rees, Astrophys. J. 620, 69 (2005).

[12] D. C. Wilkins, Phys. Rev. D 5, 814 (1972). 
[13] J. M. Bardeen, W.H. Press, and S.A. Teukolsky, Astrophys. J. 178, 347 (1972).

[14] S. A. Hughes, Phys. Rev. D 63, 064016 (2001).

[15] K.S. Thorne, R.H. Price, and D. A. MacDonald, Black Holes: The Membrane Paradigm (Yale University Press, New Haven, CT, 1986).

[16] F. D. Ryan, Phys. Rev. D 52, R3159 (1995).

[17] S. A. Hughes, Phys. Rev. D 61, 084004 (2000).

[18] B. Carter, Phys. Rev. 174, 1559 (1968).

[19] K. S. Thorne, Astrophys. J. 191, 507 (1974).

[20] Y. Mino, Phys. Rev. D 67, 084027 (2003).

[21] S. Drasco and S. A. Hughes, Phys. Rev. D 73, 024027 (2006).

[22] N. Sago, T. Tanaka, W. Hikida, and H. Nakano, Prog. Theor. Phys. 114, 509 (2005); 115, 873 (2006).

[23] K. Glampedakis, S. A. Hughes, and D. Kennefick, Phys.
Rev. D 66, 064005 (2002).

[24] J. R. Gair and K. Glampedakis, Phys. Rev. D 73, 064037 (2006).

[25] C. W. Misner, K. S. Thorne, and J. A. Wheeler, Gravitation (Freeman, San Francisco, 1973).

[26] W. Schmidt, Classical Quantum Gravity 19, 2743 (2002).

[27] A. Ori and K. S. Thorne, Phys. Rev. D 62, 124022 (2000).

[28] S. A. Teukolsky, Astrophys. J. 185, 635 (1973).

[29] M. Sasaki and T. Nakamura, Prog. Theor. Phys. 67, 1788 (1982).

[30] F. D. Ryan, Phys. Rev. D 53, 3064 (1996).

[31] D. Kennefick and A. Ori, Phys. Rev. D 53, 4319 (1996).

[32] Y. Mino, Ph.D. thesis, Kyoto University, 1996 (unpublished).

[33] Data available at http://gmunu.mit.edu/sdrasco/snapshots/. 